\title{
THE CUTOFF PHENOMENON FOR RANDOMIZED RIFFLE SHUFFLES
}

\author{
GUAN-YU CHEN AND LAURENT SALOFF-COSTE
}

\begin{abstract}
We study the cutoff phenomenon for generalized riffle shuffles where, at each step, the deck of cards is cut into a random number of packs of multinomial sizes which are then riffled together.
\end{abstract}

\section{INTRODUCTION}

In this article we consider some generalizations of the standard riffle shuffle of Gilbert, Shannon and Reeds (GSR-shuffle for short). The GSR-shuffle models the way typical card players shuffle cards. First, the deck is cut into two packs according to an $\left(n, \frac{1}{2}\right)$-binomial random variable where $n$ is the number of cards in the deck. Next, cards are dropped one by one from one or the other pack with probability proportional to the relative sizes of the packs. Hence, if the left pack contains $a$ cards and the right pack $b$ cards, the next card drops from the left pack with probability $a /(a+b)$.

The history of this model is described in [8, Chap. 4D] where the reader will also find other equivalent definitions and a discussion of how the model relates to real life card shuffling. The survey [10] gives pointers to the many developments that arose from the study of the GSR model.

Early results concerning the mixing time (i.e., how many shuffles are needed to mix up the deck) are described in [1, 2, 8. In particular, using ideas of Reeds, Aldous proved in [1] that, asymptotically as the number $n$ of cards tends to infinity, it takes $\frac{3}{2} \log _{2} n$ shuffles to mix up the deck if convergence is measured in total variation (we use $\log _{a}$ to denote base $a$ logarithms and log for natural, i.e., base $e$, logarithms).

In 4, Bayer and Diaconis obtained an exact useful formula for the probability distribution describing the state of the deck after $k$ GSR-shuffles. Namely, suppose that cards are numbered 1 through $n$ and that we start with the deck in order. Let $\sigma$ denote a given arrangement of the cards and let $Q_{n}^{k}(\sigma)$ be the probability that the deck is in state $\sigma$ after $k$ GSR-shuffles. Then

$$
Q_{n}^{k}(\sigma)=2^{-k n}\left(\begin{array}{c}
n+2^{k}-r \\
n
\end{array}\right)
$$

where $r$ is the number of rising sequences in $\sigma$. Given an arrangement of the deck, a rising sequence is a maximal subset of cards consisting of successive face values displayed in order. For instance, the arrangement $3,1,4,5,7,2,8,9,6$ has rising

1991 Mathematics Subject Classification. 60J05.

Key words and phrases. Cutoff phenomenon, riffle shuffle.

The first author is partially supported by NCTS, Taiwan.

Research partially supported by NSF Grants DMS 0102126, 0306194 and 0603886. 
sequences $(1,2),(3,4,5,6),(7,8,9)$. See [2, 4] for details. By definition, the total variation distance between two probability measures $\mu, \nu$ on a set $S$ is given by

$$
\|\mu-\nu\|_{\mathrm{TV}}=\sup _{A \subset S}\{\mu(A)-\nu(A)\}
$$

Using the formula displayed in (1.1), Bayer and Diaconis gave a very sharp version of the fact that the total variation mixing time is $\frac{3}{2} \log _{2} n$ for the GSR-shuffle.

Theorem 1.1 (Bayer and Diaconis [4]). Fix $c \in(-\infty,+\infty)$. For a deck of $n$ cards, the total variation distance between the uniform distribution and the distribution of a deck after $k=\frac{3}{2} \log _{2} n+c$ GSR-shuffles is

$$
\frac{1}{\sqrt{2 \pi}} \int_{-2^{-c} / 4 \sqrt{3}}^{2^{-c} / 4 \sqrt{3}} e^{-t^{2} / 2} d t+O_{c}\left(n^{-1 / 4}\right) .
$$

This result illustrates beautifully the so-called cutoff phenomenon discussed in [1, 2, 3, 8, 9, 14, 17. Namely, there is a sharp transition in convergence to stationarity. Indeed, the integral above becomes small very fast as $c$ tends to $+\infty$ and gets close to 1 even faster as $c$ tends to $-\infty$.

The aim of the present paper is to illustrate further the notion of cutoff using some generalizations of the GSR-shuffle. Along this way we will observe several phenomena that have not been, to the best of our knowledge, noticed before. For a deck of $n$ cards and a given integer $m$, a $m$-riffle shuffle is defined as follows. Cut the deck into $m$ packs whose sizes $\left(a_{1}, \ldots, a_{m}\right)$ form a multinomial random vector. In other words, the probability of having packs of sizes $a_{1}, \ldots, a_{m}$ is $m^{-n} \frac{n !}{a_{1} ! \ldots a_{m} !}$. Then form a new deck by dropping cards one by one from these packs with probability proportional to the relative sizes of the packs. Thus, if the packs have sizes $\left(b_{1}, \ldots, b_{m}\right)$ then the next card will drop from pack $i$ with probability $b_{i} /\left(b_{1}+\cdots+b_{m}\right)$. We will refer to an $m$-riffle shuffle simply as an $m$-shuffle in what follows. Obviously the GSR-shuffle is the same as a 2-shuffle. A 1-shuffle leaves the deck unchanged.

These shuffles were considered in [4] where the following two lemmas are proved.

Lemma 1.2. In distribution, an $m$-shuffle followed by an independent $m^{\prime}$-shuffle equals an $\mathrm{mm}^{\prime}$-shuffle.

Lemma 1.3. For a deck of $n$ cards in order, the probability that after an $m$-shuffle the deck is in state $\sigma$ depends only of the number $r=r(\sigma)$ of rising sequences of $\sigma$ and equals $Q_{n, m}(r)$ where

$$
Q_{n, m}(r)=m^{-n}\left(\begin{array}{c}
n+m-r \\
n
\end{array}\right) .
$$

For instance, formula (1.1) for the distribution of the deck after $k$ GSR-shuffles follows from a direct application of these two lemmas since $k$ consecutive independent 2 -shuffles equal a $2^{k}$-shuffle in distribution. These lemmas will play a crucial role in this paper as well.

The model we consider is as follows. Let $p=(p(1), p(2), \ldots)$ be the probability distribution of an integer valued random variable $X$, i.e.,

$$
P(X=k)=p(k), \quad k=1,2, \ldots
$$

A $p$-shuffle proceeds by picking an integer $m$ according to $p$ and performing an $m$-shuffle. In other words, the distribution of a $p$-shuffle is the $p$-mixture of the $m$-shuffle distributions. Note that casinos use multiple decks for some games and 
that these are shuffled in various ways (including by shuffling machines). The model above (for some appropriate $p$ ) is not entirely unrealistic in this context.

Because of Lemma 1.3. the probability that starting from a deck in order we obtain a deck in state $\sigma$ depends only on the number of rising sequences in $\sigma$ and is given by

$$
Q_{n, p}(r)=\sum_{1}^{\infty} p(m) Q_{n, m}(r)=E\left(Q_{n, X}(r)\right) .
$$

Abusing notation, if $\sigma$ denotes a deck arrangement of $n$ cards with $r$ rising sequences, we write

$$
Q_{n, p}(\sigma)=Q_{n, p}(r)
$$

Very generally, if $Q$ is a probability measure on deck arrangements (hence describes a shuffling method), we denote by $Q^{k}$ the distribution of the deck after $k$ such shuffles, starting from a deck in order. For instance, Lemma 1.2 yields

$$
Q_{n, m}^{k}=Q_{n, m^{k}} .
$$

Let $U_{n}$ be the uniform distribution on the set of deck arrangements of $n$ cards. Although this will not really play a role in this work, recall that deck arrangements can be viewed as elements of the symmetric group $S_{n}$ in such a way that $Q^{k}$, the distribution after $k$ successive $Q$-shuffles, is the $k$-fold convolution of $Q$ by itself. See, e.g., 1, 4, 8, 15. Each of the measures $Q_{n, p}$ generates a Markov chain on deck arrangements (i.e., on the symmetric group $S_{n}$ ) whose stationary distribution is $U_{n}$. These chains are ergodic if $p$ is not concentrated at 1 . They are not reversible. Note that [1] studies a similar but different model based on top $m$ to random shuffles. See [11, Section 2].

The goal of this paper is to study the convergence of $Q_{n, p}^{k}$ to the uniform distribution in total variation as $k$ tends to infinity and, more precisely, the occurrence of a total variation cutoff for families of shuffles $\left\{\left(S_{n}, Q_{n, p_{n}}, U_{n}\right)\right\}_{1}^{\infty}$ as the number $n$ of cards grows to infinity and $p_{n}$ is a fixed sequence of probability measures on the integers. To illustrate this, we state the simplest of our results.

Theorem 1.4. Let $p$ be a probability measure on the positive integers such that

$$
\mu=\sum_{1}^{\infty} p(k) \log k<\infty .
$$

Fix $\epsilon \in(0,1)$. Then, for any $k_{n}>(1+\epsilon) \frac{3}{2 \mu} \log n$, we have

$$
\lim _{n \rightarrow \infty}\left\|Q_{n, p}^{k_{n}}-U_{n}\right\|_{\mathrm{TV}}=0
$$

whereas, for $k_{n}<(1-\epsilon) \frac{3}{2 \mu} \log n$,

$$
\lim _{n \rightarrow \infty}\left\|Q_{n, p}^{k_{n}}-U_{n}\right\|_{\mathrm{TV}}=1 .
$$

In words, this theorem establishes a total variation cutoff at time $\frac{3}{2 \mu} \log n$ (see the definition of cutoff in Section 2 below). If $p$ is concentrated at 2, i.e., $Q_{n, p}$ represents a GSR-shuffle, then $\mu=\log 2$ and $\frac{3}{2 \mu} \log n=\frac{3}{2} \log _{2} n$ in accordance with the results of Aldous [1] and Bayer-Diaconis [4] (e.g., Theorem 1.1).

The results we obtain are more general and more precise than Theorem 1.4 in several directions. First, we will consider the case where the probability distribution $p=p_{n}$ depends on the size $n$ of the deck. This is significant because we will not 
impose that the sequence $p_{n}$ converges as $n$ tends to infinity. Second, and this may be a little surprising at first, (1.3) is not necessary for the existence of a cutoff and we will give sufficient conditions that are weaker than (1.3). Third, under stronger moment assumptions, we will describe the optimal window size of the cutoff. For instance, Theorem 1.1 says that, for the GSR-shuffle, the window size is of order 1 with a normal shape. This result generalizes easily to any $m$-shuffle where $m$ is a fixed integer greater or equal to 2. See Remark 3.1 and Theorem 5.4 below. Suppose now that instead of the GSR-shuffle we consider the $p$-shuffle with $p(2)=p(3)=1 / 2$. In this case, $\mu=\log \sqrt{6}$. Theorem 1.4 gives a total variation cutoff at time $\frac{3}{2} \log _{\sqrt{6}} n$. We will show that this cutoff has optimal window size of order $\sqrt{\log n}$. Thus picking at random between 2 and 3 shuffles changes the window size significantly when compared to either pure 2-shuffles or pure 3 -shuffles.

We close this introduction with a remark concerning the spectrum of these generalized riffle shuffles and how it relates to the window of the cutoff. As Lemma 1.2 makes clear, all riffle shuffles commute. Although riffle shuffles are not reversible, they are all diagonalizable with real positive eigenvalues and their spectra can be computed explicitly (this is another algebraic "miracle" attached to these shuffles!). See [4, 5, 6]. In particular, the second largest eigenvalue of an $m$-shuffle is $1 / m$ with the same eigenspace for all $m \geq 2$. See [13] for a stronger result implying this statement. Thus, the second largest eigenvalue of a $p$-shuffle is $\beta=\sum k^{-1} p(k)$. By definition, the relaxation time of a finite Markov chain is the inverse of the spectral gap $(1-\beta)^{-1}$ and one might expect that, quite generally, for families of Markov chains presenting a cutoff, this quantity would give a good control of the window of the cutoff. The generalized riffle shuffles studied here provided interesting (albeit non-reversible) counterexamples: Take, for instance, the case discussed earlier where $p(2)=p(3)=1 / 2$. Then $\beta=\frac{5}{12}$ and $(1-\beta)^{-1}=\frac{12}{7}$, independently of the number $n$ of cards. However, as mentioned above, the optimal window size of the cutoff for this family is $\sqrt{\log n}$. For generalized riffle shuffles, the window size of the cutoff and the relaxation time appear to be disconnected.

\section{The Cutoff Phenomenon}

The following definition introduces the notion of cutoff for a family of ergodic Markov chains.

Definition 2.1. Let $\left\{\left(S_{n}, K_{n}, \pi_{n}\right)\right\}_{1}^{\infty}$ be a family of ergodic Markov chains where $S_{n}$ denotes the state space, $K_{n}$ the Markov kernel, and $\pi_{n}$ the stationary distribution. This family satisfies a total variation cutoff with critical time $t_{n}>0$ if, for any fixed $\epsilon \in(0,1)$,

$$
\lim _{n \rightarrow \infty} \sup _{x \in S_{n}}\left\|K_{n}^{k_{n}}(x, \cdot)-\pi_{n}\right\|_{\mathrm{TV}}= \begin{cases}0 & \text { if } k_{n}>(1+\epsilon) t_{n} \\ 1 & \text { if } k_{n}<(1-\epsilon) t_{n} .\end{cases}
$$

This definition was introduced in [2]. A more thorough discussion is in [9] where many examples are described. Note that this definition does not require that the critical time $t_{n}$ tends to infinity (in [9], the corresponding definition requires that $t_{n}$ tends to infinity). The positive times $t_{n}$ can be arbitrary and thus can have several limit points in $[0, \infty]$. Examples of families having a cutoff with a bounded critical time sequence will be given below. Theorem 1.4 above states that, under assumption (1.3), a $p$-shuffle has a total variation cutoff with critical time $t_{n}=$ $\frac{3}{2 \mu} \log n$. 
Informally, a family has a cutoff if convergence to stationarity occurs in a time interval of size $o\left(t_{n}\right)$ around the critical time $t_{n}$. The size of this time interval can be thought of as the "window" of the cutoff. The next definition carefully defines the notion of the window size of a cutoff.

Definition 2.2. Let $\left\{\left(S_{n}, K_{n}, \pi_{n}\right)\right\}_{1}^{\infty}$ be a family of ergodic Markov chains as in Definition 2.1. We say that this family presents a $\left(t_{n}, b_{n}\right)$ total variation cutoff if the following conditions are satisfied:

(1) For all $n=1,2, \ldots$, we have $t_{n}>0$ and $\lim _{n \rightarrow \infty} b_{n} / t_{n}=0$.

(2) For $c \in \mathbb{R}-\{0\}$ and $n \geq 1$, set

$$
k=k(n, c)=\left\{\begin{array}{ll}
\left\lceil t_{n}+c b_{n}\right\rceil & \text { if } c>0 \\
\left\lfloor t_{n}+c b_{n}\right\rfloor & \text { if } c<0
\end{array} .\right.
$$

The functions $\bar{f}, \underline{f}$ defined by

$$
\bar{f}(c)=\limsup _{n \rightarrow \infty} \sup _{x \in S_{n}}\left\|K_{n}^{k}(x, \cdot)-\pi_{n}\right\|_{\mathrm{TV}} \text { for } c \neq 0
$$

and

$$
\underline{f}(c)=\liminf _{n \rightarrow \infty} \sup _{x \in S_{n}}\left\|K_{n}^{k}(x, \cdot)-\pi_{n}\right\|_{\mathrm{TV}} \text { for } c \neq 0
$$

satisfy

$$
\lim _{c \rightarrow \infty} \bar{f}(c)=0, \quad \lim _{c \rightarrow-\infty} \underline{f}(c)=1 .
$$

Definition 2.3. Referring to Definition 2.2, a $\left(t_{n}, b_{n}\right)$ total variation cutoff is said to be optimal if the functions $\bar{f}, \underline{f}$ satisfy $\underline{f}(c)>0$ and $\bar{f}(-c)<1$ for all $c>0$.

Note that any family having a $\left(t_{n}, b_{n}\right)$ cutoff (Definition 2.2) has a cutoff with critical time $t_{n}$ (Definition 2.1). The sequence $\left(b_{n}\right)_{1}^{\infty}$ in Definition 2.2 describes an upper bound on the optimal window size of the cutoff. For instance the main result of Bayer and Diaconis [4, i.e., Theorem 1.1 above, shows that the GSR-shuffle family presents a $\left(t_{n}, b_{n}\right)$ total variation cutoff with $t_{n}=\frac{3}{2} \log _{2} n$ and $b_{n}=1$. Theorem 1.1 actually determines exactly "the shape" of the cutoff, that is, the two functions $\bar{f}, \underline{f}$ of Definition 2.2. Namely, for the GSR-shuffle family and $t_{n}=$ $\frac{3}{2} \log _{2} n, b_{n}=1$, we have

$$
\bar{f}(c)=\underline{f}(c)=\frac{1}{\sqrt{2 \pi}} \int_{-2^{-c} / 4 \sqrt{3}}^{2^{-c} / 4 \sqrt{3}} e^{-t^{2} / 2} d t .
$$

This shows that this cut-off is optimal (Definition 2.3).

The optimality introduced in Definition 2.3 is very strong. If a family presents an optimal $\left(t_{n}, b_{n}\right)$ total variation cut-off and also a $\left(s_{n}, c_{n}\right)$ total variation cut-off, then $t_{n} \sim s_{n}$ and $b_{n}=O\left(c_{n}\right)$. In words, if $\left(t_{n}, b_{n}\right)$ is an optimal cut-off then there are no cut-offs with a window significantly smaller than $b_{n}$. For a more detailed discussion of the cutoff phenomena and their optimality, see [7].

\section{Cutoffs For Generalized RIfFle SHuffles}

In this section we state our main results and illustrate them with simple examples. They describe total variation cutoffs for generalized riffle shuffles, that is, for 
the $p$-shuffles defined in the introduction. More precisely, for each $n$ ( $n$ is the number of cards), fix a probability distribution $p_{n}=\left(p_{n}(1), p_{n}(2), \ldots\right)$ on the integers and consider the family of Markov chains (i.e., shuffles)

$$
\left\{\left(S_{n}, Q_{n, p_{n}}, U_{n}\right)\right\}_{1}^{\infty} \text {. }
$$

Here $S_{n}$ is the set of all deck arrangements (i.e., the symmetric group) and $U_{n}$ is the uniform measure on $S_{n}$. For any $x \in[0, \infty]$, set

$$
\Psi(x)=\frac{1}{\sqrt{2 \pi}} \int_{-x / 4 \sqrt{3}}^{x / 4 \sqrt{3}} e^{-t^{2} / 2} d t .
$$

We start with the simple case where the probability distributions $p_{n}$ is concentrated on exactly one integer $m_{n}$ and use the notation $Q_{n, m_{n}}$ for an $m_{n}$-shuffle.

Theorem 3.1. Let $\left(m_{n}\right)_{1}^{\infty}$ be any sequence of integers all greater than 1 and set

$$
\mu_{n}=\log m_{n}, t_{n}=\frac{3 \log n}{2 \mu_{n}} .
$$

Then the family $\left\{\left(S_{n}, Q_{n, m_{n}}, U_{n}\right)\right\}_{1}^{\infty}$ presents a $\left(t_{n}, \mu_{n}^{-1}\right)$ total variation cutoff.

Remark 3.1. When $m_{n}=m$ is constant Theorem 3.1 gives a $\left(\frac{3}{2} \log _{m} n, 1\right)$ total variation cutoff. In this case, for $k=\frac{3}{2} \log _{m} n+c$, one has the more precise result that $\left\|Q_{n, m}^{k}-U_{n}\right\|_{\mathrm{TV}}=\Psi\left(m^{-c}\right)+O_{c}\left(n^{-1 / 4}\right)$. In particular, for $m=2$, this is the Theorem of Bayer and Diaconis stated as Theorem 1.1 in the introduction.

Next we give a more explicit version of Theorem 3.1 which requires some additional notation. For any real $t>0$, set

$$
\{t\}=\left\{\begin{array}{cl}
1 / 2 & \text { if } 0<t<1 / 2 \\
k & \text { if } k-1 / 2 \leq t<k+1 / 2 \text { for some } k=1,2, \ldots,
\end{array}\right.
$$

(this is a sort of "integer part" of $t$ ) and

$$
d(t)=\left\{\begin{array}{cl}
1 / 2 & \text { if } 0<t<1 / 2 \\
t-\{t\} & \text { if } 1 / 2 \leq t<\infty .
\end{array}\right.
$$

Theorem 3.2. Let $\left(m_{n}\right)_{1}^{\infty}$ be any sequence of integers all greater than 1. Consider the family of shuffles $\left\{\left(S_{n}, Q_{n, m_{n}}, U_{n}\right)\right\}_{1}^{\infty}$ and let $\mu_{n}, t_{n}$ be as in Theorem 3.1 .

(A) Assume that $\lim _{n \rightarrow \infty} m_{n}=\infty$, that is, $\lim _{n \rightarrow \infty} \mu_{n}=\infty$. Then, we have:

(1) The family $\left\{\left(S_{n}, Q_{n, m_{n}}, U_{n}\right)\right\}_{1}^{\infty}$ always has a $\left(\left\{t_{n}\right\}, b_{n}\right)$ cutoff for any positive $b_{n}=o(1)$, that is,

$\lim _{n \rightarrow \infty} \inf _{k<\left\{t_{n}\right\}}\left\|Q_{n, m_{n}}^{k}-U_{n}\right\|_{\mathrm{TV}}=1, \lim _{n \rightarrow \infty} \sup _{k>\left\{t_{n}\right\}}\left\|Q_{n, m_{n}}^{k}-U_{n}\right\|_{\mathrm{TV}}=0$.

(2) If $\lim _{n \rightarrow \infty}\left|d\left(t_{n}\right)\right| \mu_{n}=\infty$ then there is a $\left(t_{n}, 0\right)$ cutoff, that is,

$\lim _{n \rightarrow \infty} \inf _{k \leq t_{n}}\left\|Q_{n, m_{n}}^{k}-U_{n}\right\|_{\mathrm{TV}}=1, \quad \lim _{n \rightarrow \infty} \sup _{k \geq t_{n}}\left\|Q_{n, m_{n}}^{k}-U_{n}\right\|_{\mathrm{TV}}=0$.

(3) If $\liminf _{n \rightarrow \infty}\left|d\left(t_{n}\right)\right| \mu_{n}<\infty$ then there exists a sequence $\left(n_{i}\right)_{1}^{\infty}$ tending to infinity such that

$0<\liminf _{i \rightarrow \infty}\left\|Q_{n_{i}, m_{n_{i}}}^{\left\{t_{n_{i}}\right\}}-U_{n_{i}}\right\|_{\mathrm{TV}} \leq \limsup _{i \rightarrow \infty}\left\|Q_{n_{i}, m_{n_{i}}}^{\left\{t_{n_{n}}\right\}}-U_{n_{i}}\right\|_{\mathrm{TV}}<1$.

In particular, there is no $\left(t_{n}, 0\right)$ total variation cutoff. 
(4) If $\lim _{n \rightarrow \infty} d\left(t_{n}\right) \mu_{n}=L \in[-\infty, \infty]$ exists then

$$
\lim _{n \rightarrow \infty}\left\|Q_{n, m_{n}}^{\left\lfloor\left\{t_{n}\right\}\right\rfloor}-U_{n}\right\|_{\mathrm{TV}}=\Psi\left(e^{L}\right) .
$$

(B) Assume that $\left(m_{n}\right)_{1}^{\infty}$ is bounded. Then $t_{n}$ tends to infinity, there is a $\left(t_{n}, 1\right)$ total variation cutoff and, for any fixed $k \in \mathbb{Z}$, we have

$$
0<\liminf _{n \rightarrow \infty}\left\|Q_{n, m_{n}}^{\left\{t_{n}\right\}+k}-U_{n}\right\|_{\mathrm{TV}} \leq \limsup _{n \rightarrow \infty}\left\|Q_{n, m_{n}}^{\left\{t_{n}\right\}+k}-U_{n}\right\|_{\mathrm{TV}}<1 .
$$

In particular, the $\left(t_{n}, 1\right)$ cutoff is optimal.

Example 3.1. To illustrate this result, consider the case where $m_{n}=\left\lfloor n^{\alpha}\right\rfloor$ for some fixed $\alpha>0$. In this case, we have

$$
\mu_{n} \sim \alpha \log n, t_{n}=\frac{3 \log n}{2 \mu_{n}} \sim \frac{3}{2 \alpha} \text { as } n \text { tends to infinity. }
$$

(a) Assume that $\frac{3}{2 \alpha} \in(k, k+1)$ for some $k=0,1,2, \ldots$ Then $\left|d\left(t_{n}\right)\right| \mu_{n} \rightarrow \infty$ and

$$
\lim _{n \rightarrow \infty}\left\|Q_{n, m_{n}}^{k}-U_{n}\right\|_{\mathrm{TV}}=1, \quad \lim _{n \rightarrow \infty}\left\|Q_{n, m_{n}}^{k+1}-U_{n}\right\|_{\mathrm{TV}}=0 .
$$

(b) Assume that $\frac{3}{2 \alpha}=k$ for some integer $k=1,2, \ldots$ Then $\left|d\left(t_{n}\right)\right|=O\left(n^{-\alpha}\right)$. Hence $\left|d\left(t_{n}\right)\right| \mu_{n} \rightarrow 0$ as $n$ tends to infinity. Theorem $3.2(1)$ shows that we have a $\left(k, b_{n}\right)$ cutoff where $b_{n}$ is an arbitrary sequence of positive numbers tending to 0 . That means that

$$
\lim _{n \rightarrow \infty}\left\|Q_{n, m_{n}}^{k-1}-U_{n}\right\|_{\mathrm{TV}}=1, \quad \lim _{n \rightarrow \infty}\left\|Q_{n, m_{n}}^{k+1}-U_{n}\right\|_{\mathrm{TV}}=0 .
$$

Moreover Theorem 3.2(4) gives $\lim _{n \rightarrow \infty}\left\|Q_{n, m_{n}}^{k}-U_{n}\right\|_{\mathrm{TV}}=\Psi(1)$.

Example 3.2. Consider the case where $m_{n}=\left\lfloor(\log n)^{\alpha}\right\rfloor, \alpha>0$. Then

$$
\mu_{n} \sim \alpha \log \log n, \quad t_{n} \sim \frac{3 \log n}{2 \alpha \log \log n} \text { as } n \text { tends to infinity. }
$$

Note that $t_{n}$ tends to infinity and the window size $\mu_{n}^{-1}$ goes to zero.

We now state results concerning general $p$-shuffles. We will need the following notation. For each $n$, let $p_{n}$ be a probability distribution on the integers. Let $X_{n}$ be a random variable with distribution $p_{n}$. Assume that $p_{n}$ is not supported on a single integer and set

$$
\mu_{n}=E\left(\log X_{n}\right), \quad \sigma_{n}^{2}=\operatorname{Var}\left(\log X_{n}\right), \quad \xi_{n}=\frac{\log X_{n}-\mu_{n}}{\sigma_{n}} .
$$

Consider the following conditions which may or may not be satisfied by $p_{n}$ :

$$
\begin{gathered}
\lim _{n \rightarrow \infty} \frac{\log n}{\mu_{n}}=\infty . \\
\forall \epsilon>0, \quad \lim _{n \rightarrow \infty} E\left(\xi_{n}^{2} \mathbf{1}_{\left\{\xi_{n}^{2}>\epsilon \mu_{n}^{-1} \log n\right\}}\right)=0 .
\end{gathered}
$$

Condition (3.4) should be understood as a Lindeberg type condition. We will prove in Lemma 7.1 that (3.4) implies (3.3). Example 3.5 shows that the converse is false. 
Theorem 3.3. Referring to the notation introduced above, assume that

$$
0<\mu_{n}, \sigma_{n}<\infty
$$

and set

$$
t_{n}=\frac{3 \log n}{2 \mu_{n}}, \quad b_{n}=\frac{1}{\mu_{n}} \max \left\{1, \sqrt{\frac{\sigma_{n}^{2} \log n}{\mu_{n}}}\right\} .
$$

Assume that the sequence $\left(p_{n}\right)$ satisfies (3.4). Then the family $\left\{\left(S_{n}, Q_{n, p_{n}}, U_{n}\right)\right\}_{1}^{\infty}$ presents a $\left(t_{n}, b_{n}\right)$ total variation cutoff. Moreover, if the window size $b_{n}$ is bounded from below by a positive real number, then the $\left(t_{n}, b_{n}\right)$ total variation cut-off is optimal.

Example 3.3. Assume $p_{n}=p$ is independent of $n$ and

$$
\mu=\sum_{1}^{\infty} p(k) \log k<\infty, \quad \sigma^{2}=\sum_{1}^{\infty}|\mu-\log k|^{2} p(k)<\infty .
$$

Then condition (3.4) holds and

$$
t_{n}=\frac{3}{2 \mu} \log n, \quad b_{n} \approx \sqrt{\log n}
$$

where $b_{n} \approx \sqrt{\log n}$ means that the ratio $b_{n} / \log n$ is bounded above and below by positive constants. Thus Theorem 3.3 yields an optimal $\left(\frac{3}{2 \mu} \log n, \sqrt{\log n}\right)$ total variation cutoff.

Example 3.4. Assume that $p_{n}$ is concentrated equally on two integers $m_{n}<m_{n}^{\prime}$ and write $m_{n}^{\prime}=m_{n} k_{n}^{2}$. Thus $p_{n}\left(m_{n}\right)=p_{n}\left(m_{n} k_{n}^{2}\right)=1 / 2$ and

$$
\mu_{n}=\log m_{n} k_{n}, \quad \sigma_{n}=\log k_{n} .
$$

In this case, Condition (3.4) is equivalent to (3.3), that is

$$
\mu_{n}=\log \left(m_{n} k_{n}\right)=o(\log n) .
$$

Assuming that (3.3) holds true, Theorem 3.3 yields a total variation cutoff at time

$$
t_{n}=\frac{3 \log n}{2 \log m_{n} k_{n}}
$$

with window size

$$
b_{n}=\frac{1}{\log m_{n} k_{n}} \max \left\{1, \sqrt{\frac{\left(\log k_{n}\right)^{2} \log n}{\log m_{n} k_{n}}}\right\} .
$$

For instance, assume that $m_{n}^{\prime}=m_{n}+1$ with $m_{n}$ tending to infinity. Then (3.3) becomes $\log m_{n}=o(\log n)$ and we have

$$
b_{n}=\frac{1}{\log m_{n}} \max \left\{1, \frac{(\log n)^{1 / 2}}{m_{n}\left(\log m_{n}\right)^{1 / 2}}\right\} .
$$

Specializing further to $m_{n} \approx(\log n)^{\alpha}$ with $\alpha \in(0, \infty)$ yields

and

$$
t_{n} \sim \frac{3 \log n}{2 \alpha \log \log n}
$$

$$
b_{n} \approx\left\{\begin{array}{cl}
(\log \log n)^{-1} & \text { if } \alpha \in[1 / 2, \infty) \\
(\log n)^{1 / 2-\alpha}(\log \log n)^{-3 / 2} & \text { if } \alpha \in(0,1 / 2) .
\end{array}\right.
$$


In particular, $b_{n}=o(1)$ when $\alpha \geq 1 / 2$ but tends to infinity when $\alpha \in(0,1 / 2)$. Compare with Example 3.2 above.

Regarding Theorem 3.3, one might want to remove the hypothesis of existence of a second moment concerning the random variables $\log X_{n}$. It turns out that it is indeed possible but at the price of losing control of the window of the cutoff. What may be more surprising is that one can also obtain results without assuming that the first moment $\mu_{n}$ is finite. In some cases, it might be possible to control the window size by using convergence to symmetric stable law of exponent $\alpha \in(1,2)$ but we did not pursue this here.

Theorem 3.4. Referring to the notation introduced above, assume that $\mu_{n}>0$ (including possibly $\mu_{n}=\infty$ ). Assume further that there exists a sequence $a_{n}$ tending to infinity and satisfying

$$
a_{n}=O(\log n), \quad \lim _{n \rightarrow \infty} \frac{(\log n) E Z_{n}^{2}}{a_{n}^{2} E Y_{n}}=0, \quad \lim _{n \rightarrow \infty} \frac{\log n}{E Y_{n}}=\infty,
$$

where $Y_{n}=Z_{n}=\log X_{n}$ if $\log X_{n} \leq a_{n}$, and $Y_{n}=0, Z_{n}=a_{n}$ if $\log X_{n}>a_{n}$. Then the family $\left\{\left(S_{n}, Q_{n, p_{n}}, U_{n}\right)\right\}_{1}^{\infty}$ presents a total variation cutoff with critical time

$$
t_{n}=\frac{3 \log n}{2 E Y_{n}} .
$$

Remark 3.2. In Theorem 3.4 if (3.5) holds for some sequence $\left(a_{n}\right)$ then it also holds for any sequence $\left(d a_{n}\right)$ with $d>0$. Moreover, for all $d>0$,

$$
E\left(\left(\log X_{n}\right) \mathbf{1}_{\left\{\log X_{n} \leq d a_{n}\right\}}\right) \sim E Y_{n} .
$$

This is proved in Lemma 8.2 below.

Example 3.5. Assume $p_{n}\left(\left\lfloor e^{i}\right\rfloor\right)=c_{n}^{-1} i^{-2}$ for all $1 \leq i \leq\lfloor\log n\rfloor$, where $c_{n}=$ $1+2^{-2}+3^{-2}+\cdots+(\lfloor\log n\rfloor)^{-2}$. Note that $c_{n} \rightarrow c=\pi^{2} / 6$ as $n \rightarrow \infty$. In this case, $\mu_{n} \sim c^{-1} \log \log n, \sigma_{n}^{2} \sim c^{-1} \log n$ and for $\epsilon>0$

$$
E\left[\xi_{n}^{2} \mathbf{1}_{\left\{\xi_{n}^{2}<\epsilon \mu_{n}^{-1} \log n\right\}}\right] \sim \sqrt{\frac{\epsilon}{\log \log n}} .
$$

Hence the Lindeberg type condition (3.4) does not hold and Theorem 3.3 does not apply. However, if we consider $a_{n}=\log n$ and try to apply Theorem 3.4, we have $E Y_{n}=\mu_{n} \sim c^{-1} \log \log n$ and $E Z_{n}^{2} \sim c^{-1} \log n$. This implies that (3.5) holds and yields a total variation cutoff with critical time $\frac{\pi^{2} \log n}{4 \log \log n}$.

The untruncated version of this example is $p_{n}\left(\left\lfloor e^{i}\right\rfloor\right)=p\left(\left\lfloor e^{i}\right\rfloor\right)=c^{-1} i^{-2}, i=$ $1,2, \ldots$ and $c=\pi^{2} / 6$. In this case, $\mu_{n}=\mu=\infty$. Theorem 3.4 applies with $a_{n}=\log n$ and yields a total variation cutoff with critical time $\frac{\pi^{2} \log n}{4 \log \log n}$.

We end this section with a result which is a simple corollary of Theorem 3.4 and readily implies Theorem 1.4 .

Theorem 3.5. Let $X_{n}, p_{n}, \mu_{n}$ be as above. Assume that

$$
\mu_{n}=E\left(\log X_{n}\right)=o(\log n)
$$

and that, for any fixed $\eta>0$,

$$
E\left[\left(\log X_{n}\right) \mathbf{1}_{\left\{\log X_{n}>\eta \log n\right\}}\right]=o_{\eta}\left(\mu_{n}\right) .
$$

Then the family $\left\{\left(S_{n}, Q_{n, p_{n}}, U_{n}\right)\right\}_{0}^{\infty}$ has a total variation cutoff at time $t_{n}=\frac{3 \log n}{2 \mu_{n}}$. 
Example 3.6. Suppose $p_{n}=p$ and $0<\mu_{n}=\mu<\infty$ as in Theorem 1.4. Then condition (3.6)-(3.7) are obviously satisfied. Thus Theorem 1.4 follows immediately from Theorem 3.5 as mentioned above.

Remark 3.3. Condition (3.7) holds true if $X_{n}$ satisfies the (logarithmic) moment condition that there exists $\epsilon>0$ such that

$$
\frac{E\left(\left[\log X_{n}\right]^{1+\epsilon}\right)}{(\log n)^{\epsilon}}=o\left(\mu_{n}\right) \text {. }
$$

\section{An application: Continuous-time Card shuffling}

In this section, we consider the continuous-time version of the previous card shuffling models where the waiting times between two successive shuffles are independent exponential(1) random variables. Thus, the distribution of card arrangements at time $t$ starting from the deck in order is given by the probability measure $H_{n, t}=e^{-t\left(I-Q_{n, p_{n}}\right)}$ defined by

$$
H_{n, t}(\sigma)=H_{n, t}(r)=e^{-t} \sum_{k=0}^{\infty} \frac{t^{k}}{k !} Q_{n, p_{n}}^{k}(r) \quad \text { for } \sigma \in S_{n},
$$

where $r$ is the number of rising sequences of $\sigma$.

The definition of total variation cutoff and its optimality for continuous time families is the same as in Definitions 2.1, 2.2 and 2.3 except that all times are now taken to be non-negative reals. To state our results concerning the family $\left\{\left(S_{n}, H_{n, t}, U_{n}\right)\right\}_{1}^{\infty}$ of continuous time Markov chains associated with $p_{n}$-shuffles, $n=1,2, \ldots$, we keep the notation introduced in Section 3. In particular, we set

$$
\mu_{n}=E\left(\log X_{n}\right), \quad \sigma_{n}^{2}=\operatorname{Var}\left(\log X_{n}\right), t_{n}=\frac{3 \log n}{2 \mu_{n}},
$$

where $X_{n}$ denotes a random variable with distribution $p_{n}$, and, if $\mu_{n}, \sigma_{n} \in(0, \infty)$,

$$
\xi_{n}=\frac{\log X_{n}-\mu_{n}}{\sigma_{n}}
$$

We will obtain the following theorems as corollaries of the discrete time results of Section 3. Our first result concerns the case where each $p_{n}$ is concentrated on one integer as in Theorem 3.1

Theorem 4.1. Assume that for each $n$ there is an integer $m_{n}$ such that $p\left(m_{n}\right)=1$ Then $\mu_{n}=\log m_{n}, t_{n}=\frac{3 \log n}{2 \log m_{n}}$ and the family $\mathcal{F}=\left\{\left(S_{n}, H_{n, t}, U_{n}\right)\right\}_{1}^{\infty}$ presents a total variation cutoff if and only if

$$
\lim _{n \rightarrow \infty} \frac{\log n}{\log m_{n}}=\infty
$$

Moreover, if this condition is satisfied then $\mathcal{F}$ has an optimal $\left(t_{n}, \sqrt{t_{n}}\right)$ total variation cutoff.

Compare with the discrete time result stated in Theorem 3.1 and with Example 3.1 which we now revisit.

Example 4.1. Assume that $P\left(X_{n}=\left\lfloor n^{\alpha}\right\rfloor\right)=1$ for a fixed $\alpha>0$ as in Example 3.1. According to Theorem 4.1, the continuous time family $\mathcal{F}$ does not present a total variation cutoff in this case since $\lim _{n \rightarrow \infty} \frac{\log n}{\mu_{n}}=1 / \alpha<\infty$. Recall from Example 3.1 that the corresponding discrete time family has a cutoff. 
Assume that $P\left(X_{n}=\left\lfloor(\log n)^{\alpha}\right\rfloor\right)=1$ for some fixed $\alpha>0$ as in Example 3.3. In this case, the family $\mathcal{F}$ presents a $\left(t_{n}, \sqrt{t_{n}}\right)$ total variation cutoff with $t_{n}=\frac{3 \log n}{2 \alpha \log \log n}$. Note that the window of the continuous time cutoff differs greatly from the window of the discrete time cutoff in this case.

Next we consider the general case under various hypotheses paralleling Theorems 3.3 and 3.4 .

Theorem 4.2. Consider the continuous time family $\mathcal{F}=\left\{\left(S_{n}, H_{n, t}, U_{n}\right)\right\}_{1}^{\infty}$ associated to a sequence $\left(X_{n}\right)_{1}^{\infty}$ of integer valued random variables with probability distributions $\left(p_{n}\right)_{1}^{\infty}$.

(1) Assume that $\mu_{n}, \sigma_{n} \in(0, \infty)$ for all $n \geq 1$ and that (3.4) holds. Then the family $\mathcal{F}$ presents an optimal $\left(t_{n}, b_{n}\right)$ total variation cutoff, where

$$
t_{n}=\frac{3 \log n}{2 \mu_{n}}, b_{n}=\frac{1}{\mu_{n}} \max \left\{\left(\mu_{n}+\sigma_{n}\right) \sqrt{\frac{\log n}{\mu_{n}}}, 1\right\} .
$$

(2) Assume that $\mu_{n}>0$ (including possibly $\mu_{n}=\infty$ ) and there exists a sequence $\left(a_{n}\right)_{1}^{\infty}$ tending to infinity such that (3.5) holds. Then $\mathcal{F}$ presents a total variation cutoff with critical time

$$
t_{n}=\frac{3 \log n}{2 E Y_{n}}
$$

where $Y_{n}=\left(\log X_{n}\right) \mathbf{1}_{\left\{\log X_{n} \leq a_{n}\right\}}$.

Remark 4.1. Theorem 4.2(2) applies when $p_{n}=p$ is independent of $n$ and $\mu=$ $\sum_{1}^{\infty} p(k) \log k<\infty$. In this case, the family $\mathcal{F}=\left\{\left(S_{n}, H_{n, t}, U_{n}\right)\right\}_{1}^{\infty}$ presents a total variation cutoff with critical time $t_{n}=\frac{3 \log n}{2 \mu}$ as in Theorem 1.4. If in addition we assume that $\sigma^{2}=\sum_{1}^{\infty}|\mu-\log k|^{2} p(k)<\infty$ then Theorem 4.2(1) applies and shows that $\mathcal{F}$ has a $\left(t_{n}, \sqrt{\log n}\right)$ total variation cutoff. Compare with Example 3.3

We now describe how Theorem 4.2 applies to Examples 3.4 3.5 of Section 3 ,

Example 4.2. Assume, as in Example 3.4 that $p_{n}\left(m_{n}\right)=p_{n}\left(m_{n} k_{n}^{2}\right)=1 / 2$. Assume further that $\mu_{n}=\log \left(m_{n} k_{n}\right)=o(\log n)$. Then, by Theorem 4.2(1), $\mathcal{F}$ presents a $\left(t_{n}, \sqrt{t_{n}}\right)$ total variation cutoff, where

$$
t_{n}=\frac{3 \log n}{2 \log m_{n} k_{n}} .
$$

Finally, for Example 3.5, both in truncated and untruncated cases, Theorem 4.2(2) implies that the family presents a total variation cutoff with critical time $\frac{\pi^{2} \log n}{4 \log \log n}$. However, Theorem 4.2(1) is not applicable here since, in either case, the Lindeberg type condition (3.4) has been shown failed in Example 3.5

\section{TECHNiCAL TOOLS}

Two of the main technical tools we will use have already been stated as Lemma 1.2 and 1.3 in the introduction. In particular, Lemma 1.3 gives the probability distribution describing a deck of $n$ cards after an $m$-shuffle, namely,

$$
Q_{n, m}(r)=m^{-n}\left(\begin{array}{c}
n+m-r \\
n
\end{array}\right)
$$

where $r$ is the number of rising sequences in the arrangement of the deck. The next three known lemmas give further useful information concerning this distribution. 
Lemma 5.1 (Tanny, 18]). Let $R_{n, h}$ be the number of deck arrangements of $n$ cards having $r=n / 2+h$ rising sequences, $1 \leq r \leq n$. Then, uniformly in $h$,

$$
\frac{R_{n, h}}{n !}=\frac{e^{-6 h^{2} / n}}{\sqrt{\pi n / 6}}\left(1+o\left(\frac{1}{\sqrt{n}}\right)\right)
$$

Lemma 5.2 (Bayer and Diaconis, 4, Proposition 1]). Fix $a \in(0, \infty)$. For any integers $n, m$ such that $c=c(n, m)=m n^{-3 / 2}>a$ and any $r=\frac{n}{2}+h \in\{1,2, \ldots, n\}$, we have

$$
\begin{aligned}
Q_{n, m}\left(\frac{n}{2}+h\right)=\frac{1}{n !} \exp \{ & \frac{1}{c \sqrt{n}}\left(-h+\frac{1}{2}+O_{a}\left(\frac{h}{n}\right)\right) \\
& \left.-\frac{1}{24 c^{2}}-\frac{1}{2}\left(\frac{h}{c n}\right)^{2}+O_{a}\left(\frac{1}{c n}\right)\right\}
\end{aligned}
$$

as $n$ goes to infinity.

Lemma 5.3 (Bayer and Diaconis, [4, Proposition 2]). Let $h^{*}$ be the unique integer such that $Q_{n, m}\left(\frac{n}{2}+h\right) \geq \frac{1}{n !}$ if and only if $h \leq h^{*}$. Fix $a \in(0, \infty)$. For any integers $n, m$ such that $c=c(n, m)=m n^{-3 / 2}>a$, we have

$$
h^{*}=\frac{-\sqrt{n}}{24 c}+O_{a}(1)
$$

as $n$ tends to $\infty$.

The statements of Lemmas 5.2 and 5.3 are somewhat different from the statement in Propositions 1 and 2 in 4 but the same proofs apply. The following theorem generalizes [4, Theorem 4], that is, Theorem [1.1] of the introduction. The proof, based on the three lemmas above, is the same as in [4. It is omitted.

Theorem 5.4. Fix $a \in(0, \infty)$. For any integers $n, m$ such that $c=c(n, m)=$ $m n^{-3 / 2}>a$ we have

$$
\left\|Q_{n, m}-U_{n}\right\|_{\mathrm{TV}}=\frac{1}{\sqrt{2 \pi}} \int_{-1 /(4 \sqrt{3} c)}^{1 /(4 \sqrt{3} c)} e^{-t^{2} / 2} d t+O_{a}\left(n^{-1 / 4}\right) .
$$

Theorem 5.4 provides sufficient information to obtain good upper bounds on the cutoff times of generalized riffle shuffles. It is however not sufficient to obtain matching lower bounds and study the cutoff phenomenon. The reminder of this section is devoted to results that will play a crucial role in obtaining sharp lower bounds on cutoff times for generalized riffle shuffles. It is reasonable to guess that shuffling cards with an $(m+1)$-shuffle is more efficient than shuffling cards with an $m$-shuffle . The following Proposition which is crucial for our purpose says that this intuition is correct when convergence to stationarity is measured in total variation.

Proposition 5.5. For any integers $n, m$, we have

$$
\left\|Q_{n, m+1}-U_{n}\right\|_{\mathrm{TV}} \leq\left\|Q_{n, m}-U_{n}\right\|_{\mathrm{TV}} .
$$

Proof. Let $A_{m}=\left\{\sigma \in S_{n} \mid Q_{n, m}(\sigma)<\frac{1}{n !}\right\}$ for $m \geq 1$. By Lemma [5.6 below, we have $A_{m+1} \subset A_{m}$ and $Q_{n, m}(\sigma) \leq Q_{n, m+1}(\sigma)$ for $\sigma \in A_{m+1}$. This implies

$$
\begin{aligned}
\left\|Q_{n, m}-U_{n}\right\|_{\mathrm{TV}} & =U_{n}\left(A_{m}\right)-Q_{n, m}\left(A_{m}\right) \geq U_{n}\left(A_{m+1}\right)-Q_{n, m}\left(A_{m+1}\right) \\
& \geq U_{n}\left(A_{m+1}\right)-Q_{n, m+1}\left(A_{m+1}\right)=\left\|Q_{n, m+1}-U_{n}\right\|_{\mathrm{TV}} .
\end{aligned}
$$


Lemma 5.6. For any integers, $n, m$ and $r \in\{1, \ldots, n\}$, we have:

(1) $Q_{n, m}(r) \leq Q_{n, m+1}(r)$, if $Q_{n, m}(r) \leq \frac{1}{n !}$.

(2) $Q_{n, k}(r)>\frac{1}{n !}$ for all $k \geq m$, if $Q_{n, m}(r)>\frac{1}{n !}$.

In particular, if $n, m, r$ are such that $Q_{n, m}(r) \leq \frac{1}{n !}$, then

$$
k \mapsto Q_{n, k}(r)
$$

is non-decreasing on $\{1, \ldots, m\}$.

Proof. We prove this lemma by fixing $n$ and $1 \leq r \leq n$, and considering all possible cases of $m$. For $1 \leq m<r$, the first claim holds immediately from Lemma 1.3 since $Q_{n, m}(r)=0$, and no $Q_{n, m}(r)$ satisfies the assumption of the second claim.

For $m \geq r$, consider the following map

$$
x \stackrel{f}{\longmapsto} n \log \left(\frac{x+1}{x}\right)+\log \left(\frac{x-r+1}{x-r+1+n}\right) \forall x \in[r, \infty) .
$$

The formula of the distribution of deck arrangements in Lemma 1.3 implies

$$
f(m)=\log \left(\frac{Q_{n, m}(r)}{Q_{n, m+1}(r)}\right) .
$$

A direct computation on the derivative of $f$ shows that

$$
f^{\prime}(x)=\frac{n[(2 r-n-1) x-(r-1)(r-1-n)]}{x(x+1)(x-r+1)(x-r+1+n)} .
$$

Here we consider all possible relation between $r$ and $n$. If $r, n$ satisfy $\frac{n+1}{2} \leq r \leq$ $n$, then the derivative $f^{\prime}$ is positive on $[r, \infty)$. This implies that $f(x)$ is strictly increasing for $x \geq r$. As

$$
\lim _{x \rightarrow \infty} f(x)=0,
$$

it follows that the function $f$ is negative for $x \geq r$ and hence $Q_{n, m}(r) \leq Q_{n, m+1}(r)$ for $m \geq r$. This proves the first claim. Moreover, as

$$
\lim _{m \rightarrow \infty} Q_{n, m}(r)=\frac{1}{n !},
$$

we have $Q_{n, m}(r) \leq \frac{1}{n !}$ for all $m \geq r$ and $\frac{n+1}{2} \leq r \leq n$.

If $r, n$ satisfy $1 \leq r<\frac{n+1}{2}$, let $x_{0}=\frac{(r-1)(r-1-n)}{2 r-n-1}$. In this case, the derivative $f^{\prime}$ satisfies

$$
f^{\prime}(x) \begin{cases}\geq 0 & \text { if } r \leq x \leq x_{0} \\ <0 & \text { if } x>x_{0}\end{cases}
$$

This implies that $f$ is either decreasing on $[r, \infty)$ or increasing on $\left[r, x_{0}\right]$ and decreasing on $\left(x_{0}, \infty\right)$ according to whether $x_{0}<r$ or $x_{0} \geq r$.

On one hand, if $x_{0}<r$, that is, $f$ is decreasing on $[r, \infty)$, then (5.1) implies that $f$ is positive on $[r, \infty)$, which means, in particular, that $Q_{n, m}(r) \geq Q_{n, m+1}(r)$ for $m \geq r$. In this case, (5.2) implies that $Q_{n, m}(r) \geq \frac{1}{n !}$ for $m \geq r$.

On the other hand, if $x_{0} \geq r$, that is, $f$ increases on $\left[r, x_{0}\right)$ and decreases on $\left[x_{0}, \infty\right)$, then (5.1) implies that $f$ has at most one zero in $[r, \infty)$. If $f$ has no zero, then $f$ is positive on $[r, \infty)$ and thus (by (5.2)

$$
Q_{n, m}(r) \geq Q_{n, m+1}(r) \geq \frac{1}{n !} \quad \forall m \geq r,
$$

This proves claim (2) (claim (1) is empty in this case). 
If $f$ has a zero, say $z$, then (5.1) implies that $f<0$ on $[r, z)$ and $f>0$ on $(z, \infty)$. By writing $z=\lfloor z\rfloor+\epsilon$ with $\epsilon \in[0,1)$, it is easy to check that

$$
Q_{n,\lfloor z\rfloor}(r)=Q_{n,\lfloor z\rfloor+1}(r)>Q_{n, m}(r), \forall m \geq r, m \notin\{\lfloor z\rfloor,\lfloor z\rfloor+1\},
$$

when $\epsilon=0$, and

$$
Q_{n,\lfloor z\rfloor+1}(r)>Q_{n, m}(r), \forall m \geq r, m \neq\lfloor z\rfloor+1,
$$

when $\epsilon \in(0,1)$. Moreover, if we set $m_{0}=\lfloor z\rfloor+1$, then the map $m \mapsto Q_{n, m}(r)$ is increasing on $\left[r, m_{0}\right]$ and strictly decreasing on $\left[m_{0}, \infty\right)$. In the region $\left[m_{0}, \infty\right)$, (5.2) implies as before that $Q_{n, m}(r)>\frac{1}{n !}$ for $m \geq m_{0}$. In the region $\left[r, m_{0}\right]$, let $m_{1} \geq r$ be the largest integer $m$ such that $Q_{n, m}(r) \leq \frac{1}{n !}$. Then the monotonicity of the map $m \mapsto Q_{n, m}(r)$ implies that $Q_{n, m}(r) \leq \frac{1}{n !}$ for $r \leq m \leq m_{1}$ and $Q_{n, m}(r)>\frac{1}{n !}$ for $m_{1}<m \leq m_{0}$. This proves the desired inequalities.

Lemma 5.7. Consider all deck arrangements of a deck of $n$ cards.

(1) For $1 \leq r \leq n$, let $A_{r}$ be the set of deck arrangements with at least $r$ rising sequences. Then for all integers $n, m$ and $r \in\{1, \ldots, n\}$, we have

$$
U_{n}\left(A_{r}\right)-Q_{n, m}\left(A_{r}\right) \geq 0 \text {. }
$$

(2) Fix $a>0$. For integers $n, m$, let $c=c(n, m)=m n^{-3 / 2}>a$. Let $B_{c}$ be the set of deck arrangements with number of rising sequences in $\left[\frac{n}{2}-\frac{\sqrt{n}}{24 c}+n^{\frac{1}{4}}, n\right]$. Then

$$
\inf _{k \leq m}\left(U_{n}\left(B_{c}\right)-Q_{n, k}\left(B_{c}\right)\right)=\frac{1}{\sqrt{2 \pi}} \int_{-1 /(4 \sqrt{3} c)}^{1 /(4 \sqrt{3} c)} e^{-t^{2} / 2} d t+O_{a}\left(n^{-\frac{1}{4}}\right) .
$$

Proof. As $Q_{n, m}(r)$ is non-increasing in $r$, we have either $Q_{n, m}(\sigma) \leq \frac{1}{n !}$ for all $\sigma \in A_{r}$ or $Q_{n, m}(\sigma) \geq \frac{1}{n !}$ for all $\sigma \in S_{n}-A_{r}$. The inequality stated in (1) thus follows from the obvious identity

$$
U_{n}\left(A_{r}\right)-Q_{n, m}\left(A_{r}\right)=Q_{n, m}\left(S_{n}-A_{r}\right)-U_{n}\left(S_{n}-A_{r}\right) .
$$

To prove (2), let $h_{0}=-\frac{\sqrt{n}}{24 c}+n^{\frac{1}{4}}$. By Lemma 5.3 since $h_{0} \geq h^{*}$ for large $n$, we have $Q_{n, m}(\sigma) \leq \frac{1}{n !}$ for $\sigma \in B_{c}$. Lemma [5.6 then implies

$$
\inf _{k \leq m}\left(U_{n}\left(B_{c}\right)-Q_{n, k}\left(B_{c}\right)\right)=U_{n}\left(B_{c}\right)-Q_{n, m}\left(B_{c}\right) \quad \text { for } n \text { large. }
$$

By Lemmas 5.1 5.3, we have

$$
\begin{aligned}
& \left|\left(U_{n}\left(B_{c}\right)-Q_{n, m}\left(B_{c}\right)\right)-\left\|Q_{n, m}-U_{n}\right\|_{\mathrm{TV}}\right| \leq \sum_{h=h^{*}}^{h_{0}} \frac{R_{n, h}}{n !} \\
= & \frac{1}{\sqrt{2 \pi}} \int_{h^{*} \sqrt{12 / n}}^{h_{0} \sqrt{12 / n}} e^{-t^{2} / 2} d t+O\left(n^{-\frac{1}{2}}\right)=O_{a}\left(n^{-\frac{1}{4}}\right) .
\end{aligned}
$$

The equality in (2) then follows from Theorem 5.4 


\section{Proof of Theorem 3.1 , 3.2}

The following lemma is a corollary of Theorem 5.4. It is the main tool used to prove Theorems 3.1 and 3.2 .

Lemma 6.1. For $n \in \mathbb{N}$, let $m_{n} \in \mathbb{N}$ and $c_{n}=m_{n} n^{-3 / 2}$. Set

$$
\liminf _{n \rightarrow \infty} c_{n}=L, \limsup _{n \rightarrow \infty} c_{n}=U .
$$

(1) If $L>0$ (including possibly the infinity), then

$$
\limsup _{n \rightarrow \infty}\left\|Q_{n, m_{n}}-U_{n}\right\|_{\mathrm{TV}} \leq \Psi\left(L^{-1}\right) .
$$

(2) If $U<\infty$ (including possibly 0$)$, then

$$
\liminf _{n \rightarrow \infty}\left\|Q_{n, m_{n}}-U_{n}\right\|_{\mathrm{TV}} \geq \Psi\left(U^{-1}\right) .
$$

(3) If $U=L \in[0, \infty]$, then

$$
\lim _{n \rightarrow \infty}\left\|Q_{n, m_{n}}-U_{n}\right\|_{\mathrm{TV}}=\Psi\left(U^{-1}\right) .
$$

Proof. Note that (3) follows immediately from (1) and (2). As the proofs of (1) and (2) are similar, we only prove (1). Assume first that $0<L<\infty$. Let $\epsilon \in(0, L)$ and choose $N=N(\epsilon)$ such that $c_{n} \geq L-\epsilon$ for $n \geq N$. This implies that for $n \geq N$,

$$
\begin{aligned}
\left\|Q_{n, m_{n}}-U_{n}\right\|_{\mathrm{TV}} & \leq \sup _{k \geq(L-\epsilon) n^{3 / 2}}\left\|Q_{n, k}-U_{n}\right\|_{\mathrm{TV}} \\
& =\Psi\left((L-\epsilon)^{-1}\right)+O_{L}\left(n^{-1 / 4}\right),
\end{aligned}
$$

where the last equality follows from Theorem 5.4. Letting $n$ tend to infinity first and then $\epsilon$ to 0 gives (1).

If $L=\infty$, let $C \in(0, \infty)$ and choose $N=N(C)$ so large that $c_{n} \geq C$ if $n \geq N$. As in the previous case, for $n \geq N$,

$$
\left\|Q_{n . m_{n}}-U_{n}\right\|_{\mathrm{TV}} \leq \Psi\left(C^{-1}\right)+O_{C}\left(n^{-1 / 4}\right) .
$$

Now letting $n, C$ tend to infinity yields (1) again.

Proof of Theorem 3.1. For $n \geq 1$ and $c \in \mathbb{R}$, let $t_{n}=\frac{3 \log n}{2 \mu_{n}}$ and

$$
k=k(n, c)=\left\{\begin{array}{ll}
\left\lceil t_{n}+c \mu_{n}^{-1}\right\rceil & \text { if } c>0 \\
\left\lfloor t_{n}+c \mu_{n}^{-1}\right\rfloor & \text { if } c<0
\end{array} .\right.
$$

This implies

$$
m_{n}^{k} n^{-3 / 2}\left\{\begin{array}{ll}
\geq e^{c} & \text { if } c>0 \\
\leq e^{c} & \text { if } c<0
\end{array} .\right.
$$

Let $\bar{f}, \underline{f}$ be the functions introduced in Definition 2.2 By Lemmas 1.2 and 6.1 , we have

$$
\bar{f}(c) \leq \Psi\left(e^{-c}\right) \quad \text { if } c>0
$$

and

$$
\underline{f}(c) \geq \Psi\left(e^{-c}\right) \quad \text { if } c<0 .
$$

Letting $c$ tend respectively to $\infty$ and $-\infty$ proves Theorem 3.1 . 
Proof of Theorem 3.2. In this proof, $k$ always denotes a non-negative integer. We first assume that $m_{n}$ tends to infinity. Note that

$$
k \begin{cases}\geq t+1 / 2 & \text { if } k>\{t\} \\ \leq t-1 / 2 & \text { if } k<\{t\} \text { and } t \in[1 / 2, \infty) . \\ =0 & \text { if } k<\{t\} \text { and } t \in(0,1 / 2)\end{cases}
$$

This implies

$$
m_{n}^{k} n^{-3 / 2} \begin{cases}\geq m_{n}^{1 / 2} & \text { if } k>\left\{t_{n}\right\} \\ \leq m_{n}^{-1 / 2} & \text { if } k<\left\{t_{n}\right\} \text { and } t_{n} \geq 1 / 2 \\ =n^{-3 / 2} & \text { if } k<\left\{t_{n}\right\} \text { and } t_{n} \in(0,1 / 2)\end{cases}
$$

Theorem 3.2(1) thus follows from Lemmas 1.2 and 6.1 .

The proof of Theorem [3.2(2) is similar to the proof of (1) but depends on the observation that

which implies

$$
k\left\{\begin{array}{ll}
\geq t+|d(t)| & \text { if } k>t \\
\leq t-|d(t)| & \text { if } k<t
\end{array} \quad \text { for } k \in \mathbb{N}\right.
$$

$$
m_{n}^{k} n^{-3 / 2} \begin{cases}\geq \exp \left\{\left|d\left(t_{n}\right)\right| \mu_{n}\right\} & \text { if } k>t_{n} \\ \leq \exp \left\{-\left|d\left(t_{n}\right)\right| \mu_{n}\right\} & \text { if } k<t_{n}\end{cases}
$$

For Theorem $3.2(3)$, by assumptions

$$
\liminf _{n \rightarrow \infty}\left|d\left(t_{n}\right)\right| \mu_{n}<\infty, \lim _{n \rightarrow \infty} \mu_{n}=\infty .
$$

Thus we can choose $M>0$ and a sequence $\left(n_{i}\right)_{1}^{\infty}$ tending to infinity such that $\left|d\left(t_{n_{i}}\right)\right| \mu_{n_{i}} \leq M$ and $t_{n_{i}} \geq 1 / 2$ for all $i \geq 1$. Since $\{t\}=t-d(t)$ for $t \geq 1 / 2$, we have that for all $i \geq 1$,

$$
e^{-M} \leq m_{n_{i}}^{\left\{t_{n_{i}}\right\}} n_{i}^{-3 / 2} \leq e^{M} .
$$

By Lemmas 1.2 and 6.1 this implies that

$$
\limsup _{i \rightarrow \infty}\left\|Q_{n_{i}, m_{n_{i}}}^{\left\{t_{n_{i}}\right\}}-U_{n_{i}}\right\|_{\mathrm{TV}} \leq \Psi\left(e^{M}\right)<1,
$$

and

$$
\liminf _{i \rightarrow \infty}\left\|Q_{n_{i}, m_{n_{i}}}^{\left\{t_{n_{i}}\right\}}-U_{n_{i}}\right\|_{\mathrm{TV}} \geq \Psi\left(e^{-M}\right)>0 .
$$

For Theorem 3.2(4), if $L<\infty$, then the fact, $\lim _{n \rightarrow \infty} \mu_{n}=\infty$, implies that $t_{n} \geq 1 / 2$ for large $n$. In this case, $\left\{t_{n}\right\}=t_{n}-d\left(t_{n}\right) \in \mathbb{Z}$ and

$$
m_{n}^{\left\{t_{n}\right\}}=n^{3 / 2} e^{-d\left(t_{n}\right) \mu_{n}} .
$$

Then the desired inequality (3.2) follows from Lemmas 1.2 and 6.1

If $L=\infty$, let $\left(n_{i}\right)_{1}^{\infty}$ be a sequence such that $t_{n} \geq 1 / 2$ if and only if $n=n_{i}$ for some $i$. Observe that if $n \notin\left\{n_{i} \mid i=1,2, \ldots\right\}$, then $\left\lfloor\left\{t_{n}\right\}\right\rfloor=0$, and hence (3.2) follows immediately. For the sequence $\left(t_{n_{i}}\right)_{1}^{\infty}$, since (6.1) holds in this case, the discussion for $L<\infty$ is applicable for $t_{n_{i}}$ and hence (3.2) holds. This finishes the proof of (4).

We now assume that $\left(m_{n}\right)_{1}^{\infty}$ is bounded and let $N$ be an upper bound of $m_{n}$. The proof in this case is similar to the proof of (3) after observing that

$$
t_{n}+k-1<\left\{t_{n}\right\}+k<t_{n}+k+1,
$$


and

$$
\min \left\{2^{k-1}, N^{k-1}\right\} \leq m_{n}^{\left\{t_{n}\right\}+k} n^{-3 / 2} \leq \max \left\{2^{k+1}, N^{k+1}\right\}
$$

\section{Proof of Theorem 3.3}

We start with the following elementary but crucial lemma.

Lemma 7.1. Let $\left\{Y_{n}\right\}_{n=1}^{\infty}$ be a sequence of nonnegative random variables. Set

$$
\mu_{n}=E\left[Y_{n}\right], \quad \sigma_{n}^{2}=\operatorname{Var}\left(Y_{n}\right), \quad \xi_{n}=\frac{Y_{n}-\mu_{n}}{\sigma_{n}} .
$$

Suppose that $\left(a_{n}\right)_{n=1}^{\infty}$ is a sequence of positive numbers such that the Lindeberg type condition

$$
\forall \epsilon>0, \lim _{n \rightarrow \infty} E\left[\xi_{n}^{2} 1_{\left\{\xi_{n}^{2}>\epsilon a_{n}\right\}}\right]=0,
$$

holds. Then

$$
\lim _{n \rightarrow \infty} a_{n}=\infty \quad \text { and } \quad \lim _{n \rightarrow \infty} \frac{\sigma_{n}^{2}}{\mu_{n}^{2} a_{n}}=0 .
$$

Proof. Note that $E\left[\xi_{n}^{2} 1_{\left\{\xi_{n}^{2} \leq \epsilon a_{n}\right\}}\right] \leq \epsilon a_{n}$ for all $\epsilon>0$. By (7.1), this implies

$$
\liminf _{n \rightarrow \infty} a_{n} \geq \epsilon^{-1} E\left[\xi_{n}^{2}\right]=\epsilon^{-1} .
$$

Hence $\lim _{n \rightarrow \infty} a_{n}=\infty$. Next, fix $\epsilon>0$. As $Y_{n}$ is nonnegative, we have

$$
E\left[\xi_{n}^{2} 1_{\left\{\xi_{n}<0\right\}}\right] \leq \frac{\mu_{n}^{2}}{\sigma_{n}^{2}} \leq \frac{\sqrt{\epsilon} \mu_{n}^{2} a_{n}}{\sigma_{n}^{2}},
$$

for all $n$ large enough, and

$$
E\left[\xi_{n}^{2} 1_{\left\{0<\xi_{n} \leq \sqrt{\epsilon a_{n}}\right\}}\right] \leq \sigma_{n}^{-1} \sqrt{\epsilon a_{n}} E\left[\left(Y_{n}-\mu_{n}\right) 1_{\left\{0<\xi_{n} \leq \sqrt{\epsilon a_{n}}\right\}}\right] \leq \sqrt{\frac{\epsilon \mu_{n}^{2} a_{n}}{\sigma_{n}^{2}}} .
$$

Let $L=\liminf _{n \rightarrow \infty} \mu_{n}^{2} a_{n} / \sigma_{n}^{2} \in[0, \infty]$. Combining both inequalities and letting $n \rightarrow \infty$ imply

$$
1 \leq \sqrt{\epsilon}(L+\sqrt{L}) .
$$

Letting $\epsilon \rightarrow 0$ shows that $L=\infty$, that is, $\sigma_{n}^{2} /\left(\mu_{n}^{2} a_{n}\right) \rightarrow 0$.

Recall the generalized model of riffle shuffle defined in (1.2). For $n \geq 1$, let $p_{n}$ be the distribution of an integer-valued random variable $X_{n}$ and consider the family $\left\{\left(S_{n}, Q_{n, p_{n}}, U_{n}\right)\right\}_{1}^{\infty}$ where

$$
Q_{n, p_{n}}(\cdot)=E\left(Q_{n, X_{n}}(\cdot)\right)=\sum_{m=1}^{\infty} p_{n}(m) Q_{n, m}(\cdot) .
$$


Let $X_{n, 1}, X_{n, 2}, \ldots$ be a sequence of i.i.d. random variables sharing the same distribution as $X_{n}$. Then, for $a, k>0$,

$$
\begin{aligned}
& \left\|Q_{n, p_{n}}^{k}-U_{n}\right\|_{\mathrm{TV}} \leq \sum_{m=1}^{\infty} P\left(\prod_{i=1}^{k} X_{n, i}=m\right)\left\|Q_{n, m}-U_{n}\right\|_{\mathrm{TV}} \\
\leq & P\left(\prod_{i=1}^{k} X_{n, i} \leq n^{3 / 2} a\right)+P\left(\prod_{i=1}^{k} X_{n, i} \geq n^{3 / 2} a\right)\left(\Psi\left(a^{-1}\right)+O_{a}\left(n^{-1 / 4}\right)\right) \\
= & \left(\Psi\left(a^{-1}\right)-1\right) P\left\{\prod_{i=1}^{k} X_{n, i} \geq n^{3 / 2} a\right\}+1+O_{a}\left(n^{-1 / 4}\right),
\end{aligned}
$$

where the first inequality comes from the triangle inequality and the second inequality follows from Theorem 5.4 .

Consider the set $B_{a}$ defined in Lemma 5.7, that is, the subset of $S_{n}$ containing permutations with numbers of rising sequences in $\left[\frac{n}{2}-\frac{\sqrt{n}}{24 a}+n^{1 / 4}, n\right]$. Lemma 5.7 then implies that

$$
\begin{aligned}
& \left\|Q_{n, p_{n}}^{k}-U_{n}\right\|_{\mathrm{TV}} \geq \sum_{m \leq n^{3 / 2} a} P\left\{\prod_{i=1}^{k} X_{n, i}=m\right\}\left(U_{n}\left(B_{a}\right)-Q_{n, m}\left(B_{a}\right)\right) \\
\geq & \Psi\left(a^{-1}\right) P\left\{\prod_{i=1}^{k} X_{n, i} \leq n^{3 / 2} a\right\}+O_{a}\left(n^{-1 / 4}\right) .
\end{aligned}
$$

Proof of Theorem 3.3, For $c \in \mathbb{R}-\{0\}$, let

$$
k=k(n, c)=\left\{\begin{array}{ll}
\left\lceil t_{n}+c b_{n}\right\rceil & \text { if } c>0 \\
\left\lfloor t_{n}+c b_{n}\right\rfloor & \text { if } c<0
\end{array},\right.
$$

where $t_{n}=\frac{3 \log n}{2 \mu_{n}}$ and $b_{n}=\frac{1}{\mu_{n}} \max \left\{1, \sqrt{\frac{\sigma_{n}^{2} \log n}{\mu_{n}}}\right\}$. By hypothesis, (3.4) holds. Thus Lemma 7.1 implies

$$
\lim _{n \rightarrow \infty} t_{n}=\infty, b_{n}=o\left(t_{n}\right)
$$

By Definition 2.2. to prove a $\left(t_{n}, b_{n}\right)$ total variation cut-off, we have to show that

$$
\lim _{c \rightarrow \infty} \bar{f}(c)=0 \lim _{c \rightarrow-\infty} \underline{f}(c)=1,
$$

where

$$
\bar{f}(c)=\limsup _{n \rightarrow \infty}\left\|Q_{n, p_{n}}^{k}-U_{n}\right\|_{\mathrm{TV}}, \underline{f}(c)=\liminf _{n \rightarrow \infty}\left\|Q_{n, p_{n}}^{k}-U_{n}\right\|_{\mathrm{TV}} .
$$

Note that $b_{n} \geq \frac{1}{2 \mu_{n}}\left(1+\sqrt{\frac{\sigma_{n}^{2} \log n}{\mu_{n}}}\right)$. This implies

$$
\log \left(n^{3 / 2} e^{c / 2}\right)-k \mu_{n}+\frac{c}{2} \sqrt{\frac{\sigma_{n}^{2} \log n}{\mu_{n}}}\left\{\begin{array}{ll}
\leq 0 & \text { if } c>0 \\
\geq 0 & \text { if } c<0
\end{array} .\right.
$$

Hence, we have

$$
P\left\{\prod_{i=1}^{k} X_{n, i} \geq n^{\frac{3}{2}} e^{c / 2}\right\} \geq P\left\{\frac{\sum_{i=1}^{k} \log X_{n, i}-k \mu_{n}}{\sigma_{n} \sqrt{k}} \geq-\frac{c}{2} \sqrt{\frac{\log n}{k \mu_{n}}}\right\} \text { for } c>0
$$


and

$$
P\left\{\prod_{i=1}^{k} X_{n, i} \leq n^{\frac{3}{2}} e^{c / 2}\right\} \geq P\left\{\frac{\sum_{i=1}^{k} \log X_{n, i}-k \mu_{n}}{\sigma_{n} \sqrt{k}} \leq-\frac{c}{2} \sqrt{\frac{\log n}{k \mu_{n}}}\right\} \text { for } c<0 .
$$

For fixed $c \in \mathbb{R}-\{0\}$, consider a triangular array of random variables whose $k$-th row consists of

$$
\log X_{n, 1}, \log X_{n, 2}, \ldots, \log X_{n, k} .
$$

In this setting, $k \sim t_{n}$ and (3.4) is equivalent to the well-known Lindeberg condition for such an array. Hence the central limit theorem (e.g., [16, Theorem 1, page 329]) yields

$$
\liminf _{n \rightarrow \infty} P\left\{\prod_{i=1}^{k} X_{n, i} \geq n^{\frac{3}{2}} e^{c / 2}\right\} \geq \frac{1}{2}(1+\Psi(2 \sqrt{2} c)) \quad \text { if } c>0,
$$

and

$$
\liminf _{n \rightarrow \infty} P\left\{\prod_{i=1}^{k} X_{n, i} \leq n^{\frac{3}{2}} e^{c / 2}\right\} \geq \frac{1}{2}(1+\Psi(-2 \sqrt{2} c)) \quad \text { if } c<0 .
$$

Then, by (7.2) and (7.3), we have

$$
\bar{f}(c) \leq 1-\frac{1}{2}\left(1-\Psi\left(e^{-c / 2}\right)\right)(1+\Psi(2 \sqrt{2} c)) \text { for } c>0,
$$

and

$$
\underline{f}(c) \geq \frac{1}{2} \Psi\left(e^{-c / 2}\right)(1+\Psi(-2 \sqrt{2} c)) \quad \text { for } c<0 .
$$

Hence the $\left(t_{n}, b_{n}\right)$-cutoff is proved by letting $c$ tend to $\infty$ and $-\infty$ respectively.

For the optimality of such total variation cutoff, we need to estimate $\bar{f}(c)$ for $c<0$ and $\underline{f}(c)$ for $c>0$. Assume that $b_{n} \geq b>0$ for all $n \geq 1$. Then we have

$$
k=\left\{\begin{array}{ll}
\left\lfloor t_{n}+c b_{n}\right\rfloor>t_{n}+c b_{n}-1 \geq t_{n}+\left(c-b^{-1}\right) b_{n} & \text { if } c<0 \\
\left\lceil t_{n}+c b_{n}\right\rceil<t_{n}+c b_{n}+1 \leq t_{n}+\left(c+b^{-1}\right) b_{n} & \text { if } c>0
\end{array} .\right.
$$

Arguing as in the proof of cutoff above, we obtain

$$
\liminf _{n \rightarrow \infty} P\left\{\prod_{i=1}^{k} X_{n, i} \geq n^{\frac{3}{2}} e^{\left(c-b^{-1}\right)}\right\} \geq \frac{1}{2}\left(1-\Psi\left(4 \sqrt{2}\left(b^{-1}-c\right)\right)\right) \text { for } c<0,
$$

and

$$
\liminf _{n \rightarrow \infty} P\left\{\prod_{i=1}^{k} X_{n, i} \leq n^{\frac{3}{2}} e^{\left(c+b^{-1}\right)}\right\} \geq \frac{1}{2}\left(1-\Psi\left(4 \sqrt{2}\left(b^{-1}+c\right)\right)\right) \text { for } c>0 .
$$

Hence, the functions $\bar{f}, \underline{f}$ are bounded by

$$
\forall c<0, \bar{f}(c) \leq 1-\frac{1}{2}\left(1-\Psi\left(e^{\left(b^{-1}-c\right)}\right)\right)\left(1-\Psi\left(4 \sqrt{2}\left(b^{-1}-c\right)\right)\right)<1,
$$

and

$$
\forall c>0, \quad \underline{f}(c) \geq \frac{1}{2} \Psi\left(e^{-\left(b^{-1}+c\right)}\right)\left(1-\Psi\left(4 \sqrt{2}\left(b^{-1}+c\right)\right)\right)>0 .
$$

By Definition 2.3, the family $\left\{\left(S_{n}, Q_{n, p_{n}}, U_{n}\right)\right\}_{1}^{\infty}$ has an optimal $\left(t_{n}, b_{n}\right)$ total variation cutoff. 


\section{Proof of Theorems 3.4, 3.5}

To work without assuming the existence of $\mu_{n}$, we need the following weak law of large numbers for triangular arrays. See, e.g., [12].

Theorem 8.1. (Weak law of large numbers) For each $n$, let $W_{n, k}, 1 \leq k \leq n$, be independent. Let $b_{n}>0$ with $b_{n} \rightarrow \infty$, and $\bar{W}_{n, k}=W_{n, k} 1_{\left\{\left|W_{n, k}\right| \leq b_{n}\right\}}$. Suppose that (1) $\sum_{k=1}^{n} P\left\{\left|W_{n, k}\right|>b_{n}\right\} \rightarrow 0$, and

(2) $b_{n}^{-2} \sum_{k=1}^{n} E \bar{W}_{n, k}^{2} \rightarrow 0$ as $n \rightarrow \infty$.

If we set $S_{n}=W_{n, 1}+\ldots+W_{n, n}$ and put $s_{n}=\sum_{k=1}^{n} E \bar{W}_{n, k}$, then

$$
\frac{S_{n}-s_{n}}{b_{n}} \rightarrow 0 \quad \text { in probability. }
$$

Proof of Theorem 3.4, For $0<|\epsilon|<1$, let

$$
k=k(n, \epsilon)=\left\{\begin{array}{ll}
\left\lceil(1+\epsilon) t_{n}\right\rceil & \text { if } \epsilon>0 \\
\left\lfloor(1+\epsilon) t_{n}\right\rfloor & \text { if } \epsilon<0
\end{array} .\right.
$$

By (7.2) and (7.3), to prove a total variation cutoff with critical time $t_{n}$, it suffices to prove that for all $a>0$

$$
\lim _{n \rightarrow \infty} P\left\{\prod_{i=1}^{k} X_{n, i} \geq n^{\frac{3}{2}} a\right\}=1, \text { if } \epsilon>0,
$$

and

$$
\lim _{n \rightarrow \infty} P\left\{\prod_{i=1}^{k} X_{n, i} \leq n^{\frac{3}{2}} a\right\}=1, \text { if } \epsilon<0 .
$$

Indeed, if these limits holds true then (7.2) and (7.3) give

$$
\limsup _{n \rightarrow \infty}\left\|Q_{n, p_{n}}^{k}-U_{n}\right\|_{\mathrm{TV}} \leq \Psi\left(a^{-1}\right) \quad \text { for } \epsilon>0
$$

and

$$
\liminf _{n \rightarrow \infty}\left\|Q_{n, p_{n}}^{k}-U_{n}\right\|_{\mathrm{TV}} \geq \Psi\left(a^{-1}\right) \quad \text { for } \epsilon<0 .
$$

The total variation cutoff is then proved by letting $a$ tend to infinity and 0 respectively.

To prove (8.1)- (8.2), note that $E Z_{n}^{2}=E Y_{n}^{2}+a_{n}^{2} P\left\{\log X_{n}>a_{n}\right\}$. By the second part of assumption (3.5), we have

$$
(1+\epsilon) t_{n} P\left\{\log X_{n}>a_{n}\right\} \rightarrow 0 \quad \text { and } \quad(1+\epsilon) t_{n} a_{n}^{-2} E Y_{n}^{2} \rightarrow 0, \quad \text { as } n \rightarrow \infty .
$$

In order to apply Theorem 8.1, for fixed $\epsilon \in(-1,1)$, consider

$$
W_{k, 1}=\log X_{n, 1}, \ldots, W_{k, k}=\log X_{n, k}
$$

as the $k$-th row of a triangular array of random variables. Then (8.3) shows that the hypotheses (1) and (2) in Theorem 8.1 hold. Hence

$$
a_{n}^{-1}\left(\sum_{i=1}^{k} \log X_{n, i}-(1+\epsilon) t_{n} E Y_{n}\right) \rightarrow 0 \text { in probability. }
$$


Note also that for $a>0, a_{n}^{-1}\left(\log \left(n^{3 / 2} a\right)-(1+\epsilon) t_{n} E Y_{n}\right) \sim \frac{-3 \epsilon \log n}{2 a_{n}}$. Hence the first part of assumption (3.5) implies that

$$
\begin{aligned}
& \limsup _{n \rightarrow \infty} a_{n}^{-1}\left(\log \left(n^{3 / 2} a\right)-(1+\epsilon) t_{n} E Y_{n}\right)<0 \quad \text { if } \epsilon>0, \\
& \liminf _{n \rightarrow \infty} a_{n}^{-1}\left(\log \left(n^{3 / 2} a\right)-(1+\epsilon) t_{n} E Y_{n}\right)>0 \quad \text { if } \epsilon<0 .
\end{aligned}
$$

Combining both (8.4) and (8.5) proves (8.1) and (8.2).

Proof of Theorem 3.5. Let $X_{n}$ be integer valued random variables such that

$$
P\left\{X_{n}=k\right\}=p_{n}(k) \quad \text { for } k=1,2, \ldots
$$

and satisfying (3.6), (3.7). Let $a_{n}=\log n$ in Theorem 3.4 so that

$$
Y_{n}=(\log X) \mathbf{1}_{\{\log X \leq \log n\}}, Z_{n}=Y_{n}+(\log n) \mathbf{1}_{\{\log X>\log n\}} .
$$

Set $L_{n}=\log X_{n}$. By (3.7), we have $E\left(L_{n} \mathbf{1}_{\left\{L_{n}>\log n\right\}}\right)=o\left(\mu_{n}\right)$. Hence $E\left(Y_{n}\right) \sim \mu_{n}$ and the third condition of (3.5) follows from (3.6). To apply Theorem 3.4, it remains to show

$$
\lim _{n \rightarrow \infty} \frac{E Y_{n}^{2}}{E Y_{n} \log n}=0, \quad \lim _{n \rightarrow \infty} \frac{P\left\{L_{n}>\log n\right\} \log n}{E Y_{n}}=0,
$$

or equivalently,

$$
\lim _{n \rightarrow \infty} \frac{E Y_{n}^{2}}{\mu_{n} \log n}=0, \quad \lim _{n \rightarrow \infty} \frac{P\left\{L_{n}>\log n\right\} \log n}{\mu_{n}}=0 .
$$

The hypothesis (3.7) gives

$$
\frac{P\left\{L_{n}>\log n\right\} \log n}{\mu_{n}} \leq \frac{E\left(L_{n} \mathbf{1}_{\left\{L_{n}>\log n\right\}}\right)}{\mu_{n}}=o(1)
$$

which proves the second desired limit. For the first limit, for any $\eta \in(0,1)$, write

$$
\begin{aligned}
E Y_{n}^{2} & =E\left[L_{n}^{2} \mathbf{1}_{\left\{L_{n} \leq \eta \log n\right\}}\right]+E\left(L_{n}^{2} \mathbf{1}_{\left\{\eta \log n<L_{n} \leq \log n\right\}}\right) \\
& \leq \eta \mu_{n} \log n+E\left(L_{n} \mathbf{1}_{\left\{L_{n}>\eta \log n\right\}}\right) \log n \\
& \leq\left(\eta+o_{\eta}(1)\right) \mu_{n} \log n
\end{aligned}
$$

where we have used (3.7) again to obtain the last inequality. Thus

$$
\frac{E Y_{n}^{2}}{\mu_{n} \log n} \leq \eta+o_{\eta}(1)
$$

Letting $n$ tend to infinity and then $\eta$ tend to 0 shows that the left-hand side tends to 0 as desired.

The next lemma deals with condition (3.5) appearing in Theorem 3.4 and plays a role in the proof of Theorem $4.2(2)$.

Lemma 8.2. For $n \geq 1$, let $a_{n}, b_{n}>0$ and $X_{n}$ be a non-negative random variable. According to the sequence $\left(a_{n}\right)_{1}^{\infty}$ and $c>0$, set $Y_{n}=X_{n} \mathbf{1}_{\left\{X_{n} \leq c a_{n}\right\}}$ and $Z_{n}=$ $Y_{n}+c a_{n} \mathbf{1}_{\left\{X_{n}>c a_{n}\right\}}$. Consider the following conditions.

$$
a_{n}=O\left(b_{n}\right), \lim _{n \rightarrow \infty} \frac{b_{n} E Z_{n}^{2}}{a_{n}^{2} E Y_{n}}=0, \lim _{n \rightarrow \infty} \frac{b_{n}}{E Y_{n}}=\infty .
$$

Then (8.6) holds for some $c>0$ if and only if it holds for any $c>0$. 
Proof. On direction is obvious. For the other direction, we assume that (8.6) holds for some $c>0$. The second condition in (8.6) implies

$$
P\left\{X_{n}>c a_{n}\right\}=o\left(\frac{E Y_{n}}{b_{n}}\right), \quad \frac{E Y_{n}^{2}}{a_{n}^{2}}=o\left(\frac{E Y_{n}}{b_{n}}\right) .
$$

Let $d>0$ and $Y_{n}^{\prime}=X_{n} \mathbf{1}_{\left\{X_{n} \leq d a_{n}\right\}}$ and $Z_{n}^{\prime}=Y_{n}^{\prime}+d a_{n} \mathbf{1}_{\left\{X_{n}>d a_{n}\right\}}$. Then (8.7) and Chebyshev inequality imply

$$
\begin{aligned}
\left|E Y_{n}^{\prime}-E Y_{n}\right| & \leq \begin{cases}c a_{n} P\left\{Y_{n}>d a_{n}\right\} & \text { if } d<c \\
d a_{n} P\left\{X_{n}>c a_{n}\right\} & \text { if } d>c\end{cases} \\
& =o\left(\frac{a_{n} E Y_{n}}{b_{n}}\right)=o\left(E Y_{n}\right),
\end{aligned}
$$

and

$$
\begin{aligned}
& \left|E Z_{n}^{\prime 2}-E Z_{n}^{2}\right| \leq\left|d^{2}-c^{2}\right| a_{n}^{2} P\left\{X_{n}>(d \wedge c) a_{n}\right\} \\
= & \left|d^{2}-c^{2}\right| a_{n}^{2}\left(P\left\{Y_{n}>(d \wedge c) a_{n}\right\}+P\left\{X_{n}>c a_{n}\right\}\right) \\
\leq & \left|d^{2}-c^{2}\right| a_{n}^{2}\left(\frac{E Y_{n}^{2}}{(d \wedge c)^{2} a_{n}^{2}}+P\left\{X_{n}>c a_{n}\right\}\right)=o\left(\frac{a_{n}^{2} E Y_{n}}{b_{n}}\right) .
\end{aligned}
$$

Hence we have $E Y_{n}^{\prime} \sim E Y_{n}$ and $\frac{b_{n} E\left(Z_{n}^{\prime}\right)^{2}}{d^{2} a_{n}^{2} E Y_{n}^{\prime}} \rightarrow 0$.

\section{Proofs of Theorems 4.1 and 4.2}

In this section we are concerned with the continuous time process whose distribution at time $t, H_{n, t}$, is given by (4.1), that is

$$
H_{n, t}=e^{-t} \sum_{k=0}^{\infty} \frac{t^{k}}{k !} Q_{n, p_{n}}^{k} .
$$

Let $X_{n, 1}, X_{n, 2}, \ldots$ be a sequence of independent random variables with probability distribution $p_{n}$. Let $\tilde{X}_{n}$ be an integer valued random variable whose probability distribution $\tilde{p}_{n}$ is given by

$$
\tilde{p}_{n}(l)=P\left\{\tilde{X}_{n}=l\right\}=\left\{\begin{array}{ll}
e^{-P\left\{X_{n, 1} \neq 1\right\}} & \text { if } l=1 \\
e^{-1} \sum_{1}^{\infty} \frac{1}{j !} P\left\{\prod_{1}^{j} X_{n, i}=l\right\} & \text { if } l>1
\end{array} .\right.
$$

With this notation, we have

$$
H_{n, 1}=E\left(Q_{n, \tilde{X}_{n}}\right)=Q_{n, \tilde{p}_{n}}
$$

and

$$
H_{n, k}=E\left(Q_{n, \tilde{X}_{n}}^{k}\right)=Q_{n, \tilde{p}_{n}}^{k}, \quad k=1,2, \ldots
$$

Let $h$ be any nonnegative function defined on $[0, \infty)$ satisfying $h(0)=0$. Fubini's Theorem yields

$$
E\left(h\left(\log \tilde{X}_{n}\right)\right)=e^{-1} \sum_{j=1}^{\infty} \frac{1}{j !} E\left(h\left(\bar{X}_{n, j}\right)\right),
$$

where $\bar{X}_{n, j}=\log X_{n, 1}+\cdots+\log X_{n, j}$. Thus, if we assume that $\mu_{n}, \sigma_{n}<\infty$ and let $h(t)=t\left(\operatorname{resp} . h(t)=t^{2}\right)$, we obtain

$$
E\left(\log \tilde{X}_{n}\right)=\mu_{n} \quad \text { and } \quad \operatorname{Var}\left(\log \tilde{X}_{n}\right)=\sigma_{n}^{2}+\mu_{n}^{2} .
$$


Proof of Theorem 4.1. Here, we deal with the case where, for each $n, p_{n}\left(m_{n}\right)=1$ for some integer $m_{n}$. Observe that for any integers $n, M$ and time $t>0$,

$$
\begin{aligned}
& \left\|H_{n, t}-U_{n}\right\|_{\mathrm{TV}} \geq H_{n, t}(i d)-\frac{1}{n !} \geq e^{-t}-\frac{1}{n !} \\
& \left\|H_{n, t}-U_{n}\right\|_{\mathrm{TV}} \leq e^{-t} \sum_{i=0}^{M} \frac{t^{i}}{i !}+\left\|Q_{n, p_{n}}^{M}-U_{n}\right\|_{\mathrm{TV}},
\end{aligned}
$$

where $i d$ is the identity of $S_{n}$, that is, represents the deck in order.

Assume that

$$
\liminf _{n \rightarrow \infty} \frac{\log n}{\mu_{n}}<\infty
$$

Let $M$ be an integer and $\left(n_{k}\right)_{1}^{\infty}$ be an increasing sequence such that $\sup _{k \geq 1} \frac{2 \log n_{k}}{\mu_{n_{k}}}<$ $M$. Let $\left(t_{k}\right)_{1}^{\infty}$ be an arbitrary sequence of positive numbers. Then, by Theorem 3.1 and the observation above, we have

$$
\lim _{k \rightarrow \infty}\left\|H_{n_{k}, t_{k}}-U_{n_{k}}\right\|_{\mathrm{TV}}=0 \Longleftrightarrow \lim _{k \rightarrow \infty} t_{k}=\infty .
$$

This means that the subfamily $\left\{\left(S_{n_{k}}, H_{n_{k}, t}, U_{n_{k}}\right)\right\}_{1}^{\infty}$, and thus $\mathcal{F}$ itself, does not present a total variation cutoff.

Assume now that

$$
\lim _{n \rightarrow \infty} \frac{\log n}{\mu_{n}}=\infty
$$

Then $t_{n}=\frac{3 \log n}{2 \mu_{n}}$ tends to infinity and thus $t_{n} \sim\left\lfloor t_{n}\right\rfloor$. Clearly, a $\left(t_{n}, \sqrt{t_{n}}\right)$ cutoff for $H_{n, t}$ is equivalent to a $\left(t_{n}, \sqrt{t_{n}}\right)$ cutoff for $Q_{n, \tilde{p}_{n}}^{k}$. We now prove the desired cutoff by applying Theorem 3.3 to $Q_{n, \tilde{p}_{n}}$. To this end, we need to show that (3.4) holds for $\tilde{X}_{n}$. Set $\tilde{\xi}_{n}=\frac{\log \tilde{X}_{n}-\mu_{n}}{\sqrt{\sigma_{n}^{2}+\mu_{n}^{2}}}$. Then (9.2) implies

$$
E\left(\tilde{\xi}_{n}^{2} \mathbf{1}_{\left\{\tilde{\xi}_{n}^{2}>\epsilon \frac{\log n}{\mu_{n}}\right\}}\right)=\sum_{j>\sqrt{\frac{\epsilon \log n}{\mu_{n}}}}^{\infty} \frac{e^{-1} j^{2}}{(j+1) !} \rightarrow 0 \quad \text { as } n \rightarrow \infty,
$$

for any $\epsilon>0$ and $n \geq m_{n}^{1 / \epsilon}$. Hence (3.4) holds for $\tilde{X}_{n}$ and, by Theorem 3.3, the family $\left\{\left(S_{n}, Q_{n, \tilde{p}_{n}}, U_{n}\right)\right\}_{1}^{\infty}$ presents, as desired, an optimal $\left(t_{n}, b_{n}\right)$ total variation cutoff with $b_{n}=\sqrt{\log n / \mu_{n}}$.

Proof of Theorem 4.2(1). As in the proof of Theorem 4.1 the desired cutoff for the family $\left\{\left(S_{n}, H_{n, t}, U_{n}\right)\right\}_{1}^{\infty}$ is equivalent to the same cutoff for $\left\{\left(S_{n}, Q_{n, \tilde{p}_{n}}, U_{n}\right)\right\}_{1}^{\infty}$ because cutoff time and window size tend to infinity. Hence, the desired conclusion will follow from Theorem 3.3 if we can show that $\tilde{X}_{n}$ at (9.1) satisfies (3.4). Set $\tilde{\xi}_{n}=\frac{\log \tilde{X}_{n}-\mu_{n}}{\sqrt{\sigma_{n}^{2}+\mu_{n}^{2}}}$. Then (9.2) implies

$$
E\left(\tilde{\xi}_{n}^{2} \mathbf{1}_{\left\{\tilde{\xi}_{n}^{2}>\epsilon \frac{\log n}{\mu_{n}}\right\}}\right)=e^{-1} \sum_{j=1}^{\infty} \frac{1}{j !} E\left(\frac{\left(\bar{X}_{n, j}-\mu_{n}\right)^{2}}{\sigma_{n}^{2}+\mu_{n}^{2}} \mathbf{1}_{\left\{\frac{\left(\bar{x}_{n, j}-\mu_{n}\right)^{2}}{\sigma_{n}^{2}+\mu_{n}^{2}}>\epsilon \frac{\log n}{\mu_{n}}\right\}}\right),
$$


if $\epsilon \mu_{n}^{-1} \log n>1$. Fix $\epsilon, \delta>0$ and let $M=M(\delta) \in \mathbb{N}, N=N(\epsilon, M) \in \mathbb{N}$ such that $2 \sum_{M+1}^{\infty} \frac{j^{2}}{j !}<\delta$ and $\sqrt{\frac{\epsilon \log n}{\mu_{n}}} \geq 2 M$ if $n \geq N$. In this case, (9.3) implies that

$$
E\left(\tilde{\xi}_{n}^{2} \mathbf{1}_{\left\{\tilde{\xi}_{n}^{2}>\epsilon \frac{\log n}{\mu_{n}}\right\}}\right) \leq \delta+e^{-1} \sum_{j=1}^{M} \frac{1}{j !} E\left(\frac{\left(\bar{X}_{n, j}-\mu_{n}\right)^{2}}{\sigma_{n}^{2}+\mu_{n}^{2}} \mathbf{1}_{\left\{\frac{\bar{x}_{n, j}-\mu_{n}}{\sqrt{\sigma_{n}^{2}+\mu_{n}^{2}}}>\sqrt{\frac{\epsilon \log n}{\mu_{n}}}\right\}}\right) .
$$

To bound the expectation in the right hand side, we consider the following sets. For $1 \leq i \leq j \leq M$, let

$$
\begin{aligned}
A_{n, i, j} & =\left\{\log X_{n, i}>\frac{1}{j}\left(\mu_{n}+\sqrt{\frac{\epsilon\left(\sigma_{n}^{2}+\mu_{n}^{2}\right) \log n}{\mu_{n}}}\right)\right\} \\
B_{n, i} & =\left\{\frac{\left(\log X_{n, i}-\mu_{n}\right)^{2}}{\sigma_{n}^{2}}>\frac{\epsilon \log n}{4 M^{2} \mu_{n}}\right\} .
\end{aligned}
$$

Then

$$
\left\{\frac{\bar{X}_{n, j}-\mu_{n}}{\sqrt{\sigma_{n}^{2}+\mu_{n}^{2}}}>\sqrt{\frac{\epsilon \log n}{\mu_{n}}}\right\} \subset \bigcup_{i=1}^{j} A_{n, i, j}
$$

and

$$
A_{n, i, j} \subset B_{n, i} \quad \text { if } \sqrt{\frac{\epsilon \log n}{\mu_{n}}} \geq 2 M
$$

This implies that for $n \geq N, 1 \leq i \leq j \leq M$,

$$
\begin{aligned}
& E\left(\frac{\left(\bar{X}_{n, j}-\mu_{n}\right)^{2}}{\sigma_{n}^{2}+\mu_{n}^{2}} \mathbf{1}_{A_{n, i, j}}\right) \\
\leq & 2 E\left(\frac{\left(\bar{X}_{n, j}-\log X_{n, i}\right)^{2}}{\sigma_{n}^{2}+\mu_{n}^{2}} \mathbf{1}_{B_{n, i}}\right)+2 E\left(\frac{\left(\log X_{n, i}-\mu_{n}\right)^{2}}{\sigma_{n}^{2}} \mathbf{1}_{B_{n, i}}\right) \\
= & \frac{2\left((j-1) \sigma_{n}^{2}+(j-1)^{2} \mu_{n}^{2}\right)}{\sigma_{n}^{2}+\mu_{n}^{2}} P\left\{B_{n, i}\right\}+2 E\left(\xi_{n}^{2} \mathbf{1}_{\left\{\xi_{n}^{2}>\frac{\epsilon \log n}{4 M^{2} \mu_{n}}\right\}}\right) \\
\leq & 3 E\left(\xi_{n}^{2} \mathbf{1}_{\left\{\xi_{n}^{2}>\frac{\epsilon \log n}{4 M^{2} \mu_{n}}\right\}}\right) \text { if } n \text { is large. }
\end{aligned}
$$

Now, using (9.5) and these estimates in (9.4), and applying the hypothesis that $X_{n}$ satisfies (3.4), we obtain

$$
\limsup _{n \rightarrow \infty} E\left(\tilde{\xi}_{n}^{2} 1_{\left\{\tilde{\xi}_{n}^{2}>\epsilon \frac{\log n}{\mu_{n}}\right\}}\right) \leq \delta \quad \forall \delta, \epsilon>0 .
$$

Hence (3.4) holds for $\tilde{X}_{n}$. By Theorem 3.3. the family $\left\{\left(S_{n}, H_{n, t}, U_{n}\right)\right\}_{1}^{\infty}$ presents an optimal $\left(\frac{3 \log n}{2 \mu_{n}}, b_{n}\right)$ total variation cutoff, where

$$
b_{n}=\frac{1}{\mu_{n}} \max \left\{\sqrt{\frac{\left(\sigma_{n}^{2}+\mu_{n}^{2}\right) \log n}{\mu_{n}}}, 1\right\}
$$

(note that $b_{n}$ always tends to infinity).

Proof of Theorem 4.2(2). The proof is similar to that of part (1) except that we will use Theorem 3.4 instead of Theorem 3.3 , Let

$$
\tilde{Y}_{n}=\left(\log \tilde{X}_{n}\right) \mathbf{1}_{\left\{\log \tilde{X}_{n} \leq a_{n}\right\}}, \quad \tilde{Z}_{n}=\tilde{Y}_{n}+a_{n} \mathbf{1}_{\left\{\log \tilde{X}_{n}>a_{n}\right\}} .
$$


By (9.2), we have

$$
E \tilde{Y}_{n}=e^{-1} \sum_{j=1}^{\infty} \frac{1}{j !} E\left[\left(\sum_{i=1}^{j} \log X_{n, i}\right) \mathbf{1}_{\left\{\sum_{1}^{j} \log X_{n, i} \leq a_{n}\right\}}\right] .
$$

It is apparent that $E \tilde{Y}_{n} \leq E Y_{n}$. For $j>0$, we have

$$
\begin{aligned}
E\left[\left(\sum_{i=1}^{j} \log X_{n, i}\right) \mathbf{1}_{\left\{\sum_{1}^{j} \log X_{n, i} \leq a_{n}\right\}}\right] \geq \sum_{i=1}^{j}\{ & E\left(\log X_{n, i} \mathbf{1}_{\left\{\log X_{n, i} \leq \frac{a_{n}}{j}\right\}}\right) \\
& \left.\times \prod_{\substack{k=1 \\
k \neq i}}^{j} P\left(\log X_{n, k} \leq \frac{a_{n}}{j}\right)\right\} .
\end{aligned}
$$

By Lemma 8.2 (or Remark 3.2) and (8.7), we have

$$
\liminf _{n \rightarrow \infty} E\left[\left(\sum_{i=1}^{j} \log X_{n, i}\right) 1_{\left\{\sum_{1}^{j} \log X_{n, i} \leq a_{n}\right\}}\right] / E Y_{n} \geq j .
$$

Hence, for $k>0$

$$
\liminf _{n \rightarrow \infty} \frac{E \tilde{Y}_{n}}{E Y_{n}} \geq e^{-1} \sum_{j=0}^{k} \frac{1}{j !} .
$$

Letting $k \rightarrow \infty$ implies $E \tilde{Y}_{n} \sim E Y_{n}$.

To apply Theorem 3.4, it remains to prove that the second part of (3.5) holds for $\tilde{Y}_{n}$ and $\tilde{Z}_{n}$, that is,

$$
E\left(\tilde{Y}_{n}^{2}\right)=o\left(\frac{a_{n}^{2} E Y_{n}}{\log n}\right), \quad P\left\{\log \tilde{X}_{n}>a_{n}\right\}=o\left(\frac{E Y_{n}}{\log n}\right) .
$$

Note that, by the hypothesis that $X_{n}$ satisfies (3.5), we have

$$
E\left(Y_{n}^{2}\right)=o\left(\frac{a_{n}^{2} E Y_{n}}{\log n}\right), \quad P\left\{\log X_{n}>a_{n}\right\}=o\left(\frac{E Y_{n}}{\log n}\right) .
$$

Then (9.2), Lemma 8.2 and the above observation imply

$$
\begin{aligned}
E\left(\tilde{Y}_{n}^{2}\right) & =e^{-1} \sum_{j=1}^{\infty} \frac{1}{j !} E\left[\left(\sum_{i=1}^{j} \log X_{n, i}\right)^{2} \mathbf{1}_{\left\{\sum_{1}^{j} \log X_{n, i} \leq a_{n}\right\}}\right] \\
& \leq e^{-1} \sum_{j=1}^{\infty} \frac{1}{j !} E\left(\sum_{i=1}^{j}\left(\log X_{n, i}\right) \mathbf{1}_{\left\{\log X_{n, i} \leq a_{n}\right\}}\right)^{2} \\
& =E Y_{n}^{2}+\left(E Y_{n}\right)^{2} \leq 2 E Y_{n}^{2}=o\left(\frac{a_{n}^{2} E Y_{n}}{\log n}\right),
\end{aligned}
$$

and

$$
\begin{aligned}
P\left\{\log \tilde{X}_{n}>a_{n}\right\} & =e^{-1} \sum_{j=1}^{\infty} \frac{1}{j !} P\left\{\sum_{i=1}^{j} \log X_{n, i}>a_{n}\right\} \\
& \leq e^{-1} \sum_{j=1}^{\infty} \frac{1}{(j-1) !} P\left\{\log X_{n}>\frac{a_{n}}{j}\right\} .
\end{aligned}
$$


Since, for $j \geq 1$,

$$
\begin{aligned}
P\left\{\log X_{n}>\frac{a_{n}}{j}\right\} & =P\left\{\log X_{n}>a_{n}\right\}+P\left\{Y_{n}>\frac{a_{n}}{j}\right\} \\
& =P\left\{\log X_{n}>a_{n}\right\}+\frac{j^{2} E Y_{n}^{2}}{a_{n}^{2}}=j^{2} \times o\left(\frac{E Y_{n}}{\log n}\right),
\end{aligned}
$$

we have

$$
P\left\{\log \tilde{X}_{n}>a_{n}\right\}=o\left(\frac{E Y_{n}}{\log n}\right) .
$$

By Theorem 3.4, the family $\left\{\left(S_{n}, Q_{n, \tilde{p}_{n}}, U_{n}\right)\right\}_{1}^{\infty}$ presents a total variation cutoff with critical time $\frac{3 \log n}{2 E Y_{n}}$. Hence the same holds for $\left\{\left(S_{n}, H_{n, t}, U_{n}\right)\right\}_{1}^{\infty}$.

\section{REFERENCES}

[1] Aldous, D. Random walks on finite groups and rapidly mixing Markov chains. Seminar on probability, XVII, 243-297, Lecture Notes in Math., 986, Springer, Berlin, 1983.

[2] Aldous, D. and Diaconis, P. Shuffling cards and stopping times. Amer. Math. Monthly 93, 333-348, 1986.

[3] Aldous, D. and Diaconis, P. Strong uniform times and finite random walks. Adv. in Appl. Math. 8, 69-97, 1986.

[4] Bayer, D. and Diaconis, P. Trailing the Dovetail Shuffle to its Lair. Ann. Appl. Probab. 2 294-313, 1992.

[5] Bidigare, P., Hanlon, P. and Rockmore, D. A combinatorial description of the spectrum for the Tsetlin library and its generalization to hyperplane arrangements. Duke Math. J. 99, 135-174, 1999.

[6] Brown, K. and Diaconis, P. Random walks and hyperplane arrangements. Ann. Probab. 26, 1813-1854, 1998.

[7] Chen, G.-Y. Cutoff phenomenon for finite Markov chains. Ph.D. dissertation.

[8] Diaconis, P. representations in probability and statistics. Institute of Mathematical Statistics Lecture Notes-Monograph Series, 11. Institute of Mathematical Statistics, Hayward, CA, 1988.

[9] Diaconis, P. The cutoff phenomenon in finite Markov chains. Proc. Nat. Acad. Sci. U.S.A. 93-4, 1659-1664, 1996.

[10] Diaconis, P. Mathematical developments from the analysis of riffle shuffling. In "Groups, combinatorics \& geometry" (Durham, 2001), 73-97, World Sci. Publishing, 2003.

[11] Diaconis, P., Fill, J. and Pitman, J. Analysis of top to random shuffles. Combin. Probab. Comput. 1, 135-155, 1992.

[12] Durrett, R. Probability: Theory and Examples. Duxbury press, 2nd ed, 1995.

[13] Mahajan, S. Shuffles on Coxeter groups. Arxiv, 2001.

[14] Saloff-Coste, L. Lectures on finite Markov chains. Lectures on probability theory and statistics (Saint-Flour Summer School, 1996), 301-413, Lecture Notes in Math., 1665, Springer, Berlin, 1997.

[15] Saloff-Coste, L. Random walks on finite groups. In Probability on discrete structures, 263346, Encyclopaedia Math. Sci., 110, Springer, Berlin, 2004. (H. Kesten, ed.)

[16] Shiryaev, A.N. Probability. Springer Verlag, 2nd ed, 1996.

[17] Stark, D.; Ganesh, A. and O'Connell, N. Information loss in riffle shuffling. Combin. Probab. Comput. 11, 79-95, 2002.

[18] Tanny, S. A probabilistic interpretation of the Eulerian numbers. Duke Math. J. 40 717-722, 1973. [Correction $\mathbf{4 1}$ 689, 1974.]

Division of Mathematics, National Center for Theoretical Science, National Tsing Hua University, Hsinchu 300, Taiwan

E-mail address: gychen@math.cts.nthu.edu.tw

Cornell University, Department of Mathematics, Ithaca, NY 14853-4201

E-mail address: lsc@math.cornell.edu 